\title{
Causal Link between the Cortico-Rubral Pathway and Functional Recovery through Forced Impaired Limb Use in Rats with Stroke
}

\author{
(DAkimasa Ishida, ${ }^{1,2}$ Kaoru Isa, ${ }^{2}$ Tatsuya Umeda, ${ }^{2}$ Kazuto Kobayashi, ${ }^{3}$ Kenta Kobayashi, ${ }^{4,5}{ }^{-}$Hideki Hida, ${ }^{1}$ \\ and $\odot$ Tadashi Isa ${ }^{2,5}$ \\ ${ }^{1}$ Department of Neurophysiology and Brain Sciences, Nagoya City University Graduate School of Medical Sciences, Nagoya, 467-8601, Japan, ${ }^{2}$ Department \\ of Developmental Physiology, National Institute for Physiological Sciences, Okazaki, 444-8585, Japan, ${ }^{3}$ Department of Molecular Genetics, Institute of \\ Biomedical Sciences, Fukushima Medical University, Fukushima, 960-1295, Japan, ${ }^{4}$ Section of Viral Vector Development, National Institute for \\ Physiological Sciences, Okazaki, 444-8585, Japan, and ${ }^{5}$ Graduate University of Advanced Studies (SOKENDAI), Hayama, 240-0193, Japan
}

Intensive rehabilitation is believed to induce use-dependent plasticity in the injured nervous system; however, its causal relationship to functional recovery is unclear. Here, we performed systematic analysis of the effects of forced use of an impaired forelimb on the recovery of rats after lesioning the internal capsule with intracerebral hemorrhage (ICH). Forced limb use (FLU) group rats exhibited better recovery of skilled forelimb functions and their cortical motor area with forelimb representation was restored and enlarged on the ipsilesional side. In addition, abundant axonal sprouting from the reemerged forelimb area was found in the ipsilateral red nucleus after FLU. To test the causal relationship between the plasticity in the cortico-rubral pathway and recovery, loss-of-function experiments were conducted using a double-viral vector technique, which induces selective blockade of the target pathway. Blockade of the cortico-rubral tract resulted in deficits of the recovered forelimb function in FLU group rats. These findings suggest that the cortico-rubral pathway is a substrate for recovery induced by intensive rehabilitation after ICH.

Key words: forced limb use; intracerebral hemorrhage; red nucleus; rehabilitation; reorganization; use-dependent plasticity

\section{Significance Statement}

The research aimed at determining the causal linkage between reorganization of the motor pathway induced by intensive rehabilitative training and recovery after stroke. We clarified the expansion of the forelimb representation area of the ipsilesional motor cortex by forced impaired forelimb use (FLU) after lesioning the internal capsule with intracerebral hemorrhaging (ICH) in rats. Anterograde tracing showed robust axonal sprouting from the forelimb area to the red nucleus in response to FLU. Selective blockade of the cortico-rubral pathway by the novel double-viral vector technique clearly revealed that the increased corticorubral axonal projections had causal linkage to the recovery of reaching movements induced by FLU. Our data demonstrate that the cortico-rubral pathway is responsible for the effect of intensive limb use.

\section{Introduction}

Poststroke rehabilitation is an essential strategy to reduce the impairment of neural functions and to improve patients' quality of life. A large number of investigations have reported that reha-

Received June 24, 2015; revised Nov. 17, 2015; accepted Nov. 24, 2015.

Author contributions: A.I., H.H., and T.I. designed research; A.I. and K.I. performed research; T.U., Kazuto Kobayashi, and Kenta Kobayashi contributed unpublished reagents/analytic tools; A.I. and K.I. analyzed data; A.I. and T.I. wrote the paper.

This study was supported by a Grant-in-Aid for Scientific Research on Innovative Areas ("Adaptive Circuit Shift" to T.I., K.K., and H.H.), the Brain Mapping by Integrated Neurotechnologies for Disease Studies (Brain/MINDS) Project of the Ministry of Education, Culture, Sports, Science and Technology of Japan and Japan Agency for Medical Research and Development (T.I.), and funding for collaborative research by the National Institute for Physiological Sciences (H.H.). We thank D. Watanabe (Kyoto University) for kindly providing plasmids for viral vector production. bilitative training can induce neurophysiological and neuroanatomical plasticity in an injured CNS, such as the formation of a compensatory neuronal circuit and enhancement of synaptic plasticity (Murphy and Corbett, 2009; Dimyan and Cohen, 2011). These rehabilitation-induced plastic changes are considered crucial to the recovery of impaired limb functions (Taub et al., 1993; Jones and Schallert, 1994; Nudo et al., 1996; Biernaskie

The authors declare no competing financial interests.

Correspondence should be addressed to Tadashi Isa, Department of Developmental Physiology, National Institute for Physiological Sciences, National Institutes of Natural Sciences, Myodaiji, Okazaki, 444-8585, Japan. E-mail: tisa@nips.ac.jp.

DOI:10.1523/JNEUROSCI.2399-15.2016

Copyright $\odot 2016$ the authors $\quad 0270-6474 / 16 / 360455-13 \$ 15.00 / 0$ 
and Corbett, 2001; Murphy and Corbett, 2009; Dimyan and Cohen, 2011). Therefore, there is an urgent need to clarify the detailed relationship between the adaptive plasticity of the injured CNS and functional recovery caused by rehabilitative intervention.

Among a variety of rehabilitative training protocols, the intensive and forced use of an impaired upper limb, such as constraint-induced movement therapy (CIMT), is regarded as an effective rehabilitative strategy (Taub et al., 1993; Wolf et al., 2006). This treatment involves restraining the intact upper extremity and the forced use of the impaired extremity (Taub et al., 1993). Brain imaging studies of stroke patients indicated that CIMT induced expansion of the forelimb representative area in the sensorimotor cortex after stroke (Liepert et al., 1998; Sawaki et al., 2008). Moreover, studies using rodent models reported forced impaired limb use-induced anatomical changes of the CNS such as sprouting from the ipsilateral corticospinal tract (CST) to the denervated cervical cord (Maier et al., 2008; Zhao et al., 2013). However, it has been difficult to demonstrate the causal linkage between reorganization of the circuit and behavioral recovery. The recent development of a double-infection technique with viral vectors has enabled selective and reversible blockade of specific neural circuits (Kinoshita et al., 2012), which would promote analysis of the neural mechanism underlying the therapeutic effect of rehabilitative training. Using this technique, Wahl et al. (2014) demonstrated a causal relationship between the newly formed crossed intraspinal circuits and functional recovery in a thrombotic stroke model in rats treated with combination of rehabilitation and a novel drug therapy. In the present study, we investigated the neural mechanism of recovery induced by intensive use of an impaired forelimb, a classical rehabilitation strategy, using an ICH model in rats (Masuda et al., 2007; Ishida et al., 2011; Ueda et al., 2014; Ishida et al., 2015). The model has a small hemorrhage near the internal capsule (IC), a common site of hemorrhagic stroke (Xi et al., 2006), showing severe chronic motor deficits (Masuda et al., 2007; Ishida et al., 2011; Ishida et al., 2015).

The present study aimed at demonstrating the causal link between forced limb use (FLU)-induced plasticity of the injured neural circuits and the recovery of impaired forelimb function. We conducted longitudinal functional, electrophysiological, and successive anatomical analysis on the same ICH rats. In addition to the reorganization of the CST, the subcortical descending pathways, such as the rubrospinal and reticulospinal tracts, were considered to contribute substantially to spontaneous recovery after CNS injury (Z'Graggen et al., 1998; Ballermann and Fouad, 2006; Zaaimi et al., 2012; Esposito et al., 2014; Zörner et al., 2014; Siegel et al., 2015). In this study, the cortico-rubral pathway was shown to be responsible for the effects of FLU by loss-of-function experiments using pharmacological intervention and selective blockade of the pathway with a double-viral vector infection technique.

\section{Materials and Methods}

Animals and experimental setup. Male Wistar rats (250-300 g) were group housed under a $12 \mathrm{~h}$ light/dark cycle, with food and water available ad libitum. Figure $1 a$ shows the timeline of the performed experiments. All procedures involving animals were approved by the Institutional Animal Care and Use Committee of the National Institutes of Natural Sciences. All attempts were made to minimize the suffering and number of animals used in this study. The methods and time schedule of the present experiments are summarized in Figures $1 a$ and $5 a$.

$I C H$. The animals were anesthetized with a mixture of ketamine (70 $\mathrm{mg} / \mathrm{kg}$ body weight $)$ and xylazine $(10 \mathrm{mg} / \mathrm{kg})$. Atropine $(0.1 \mathrm{mg} / \mathrm{kg}$; Mit- subishi Tanabe Pharma) and dexamethasone $(0.1 \mathrm{mg} / \mathrm{kg}$; Banyu) were administered intramuscularly after anesthesia. Body temperature was controlled continuously at $37.0 \pm 0.5^{\circ} \mathrm{C}$. The head of the rat was fixed to a stereotaxic frame (Narishige) and the scalp was cut along the midline. A small hole was made using a drill at $3.8 \mathrm{~mm}$ lateral to the midline and 1.8 $\mathrm{mm}$ caudal to the bregma contralateral to the preferred forelimb. A total of $1.4 \mu \mathrm{l}$ of collagenase (diluted to $15 \mathrm{U} / \mathrm{ml}$ in sterile saline, type IV; Sigma-Aldrich) was injected using a glass micropipette that was connected to a Hamilton syringe $(5 \mathrm{~mm}$ below the brain surface, at a rate of $0.2 \mu \mathrm{l} / \mathrm{min}$ over $7 \mathrm{~min}$ ). The pipette and syringe were left in place for 7 min and then removed slowly. Sham-operated rats received an injection of the equivalent amount of sterile saline. After injection, the skin was sutured and the rats were returned to their home cage for recovery. To quantify the lesion extent of the IC, Klüver-Barrera staining was performed (Fig. 1c). We compared the size of the damage by measuring the area of images of remaining fibers in the IC after binarization as shown in Figure $1 d$. Percentage of the damaged area of the IC was calculated as normalized value to that of the intact IC.

FLU. FLU treatment was performed as described previously (Jones and Schallert, 1994). In brief, the rats were anesthetized by isoflurane ( $2 \%$ for induction, $1 \%$ for maintenance; DS Pharma Animal Health) and their unimpaired forelimb and upper torso were wrapped in soft felt and plaster of Paris strips. FLU group rats were forced to use their impaired forelimb in all daily activities (Fig. 1b). The upper torso of ICH rats was wrapped in the same way, but they were allowed to use both forepaws. The rats were then returned to their home cages and remained in the casts for $7 \mathrm{~d}$.

Behavioral assessments. Gross motor dysfunction was confirmed using a motor deficits score as described previously (Masuda et al., 2007; Ishida et al., 2011). In brief, this score consisted of four tests (spontaneous ipsilateral rotation, beam walking, contralateral hindlimb retraction, and bilateral forepaw grasp). Each test was designed to evaluate the degree of gross motor deficit on a 5-point scale (graded from 0 for normal to 4 for most severe dysfunction). The scores were added and expressed (maximum possible score is 16 ).

To test the skilled forelimb movements, a single-pellet reaching test was performed as described previously (Metz and Whishaw, 2000). A Plexiglas test chamber $(45 \times 13 \times 40 \mathrm{~cm})$ had a narrow slit in its front wall and a platform $(3 \mathrm{~cm}$ in width) with 2 indentations positioned at 1.5 $\mathrm{cm}$ from the center of the slit was attached to the outside of the front wall of the apparatus. The rats were placed in the chamber and trained to reach through the slit to retrieve sucrose pellets ( $45 \mathrm{mg}$; Bioserv), which was placed on either indentation diagonally to the dominant limb. Food control was performed during the period of training and testing so that the animals neither gained nor lost weight. All animals were trained for 3 weeks before surgery. Each training session consisted of 20 trials per day. If a rat failed to retrieve $>10$ pellets per session on the final 3 consecutive days of the training period, it was excluded from the study. Behavioral assessments were performed at 10-12 and 26-28 d after ICH surgery. To assess the stepping function of the forelimb, the rats were trained to cross a 1-m-long horizontal ladder with rungs spaced irregularly at a distance of $1-3 \mathrm{~cm}$ at a constant speed for $3 \mathrm{~d}$ before surgery (Metz and Whishaw, 2002). To analyze the coordinated movement of the forelimb, the rats were videotaped while crossing the ladder and the percentage of steps that slipped from the rungs was calculated. Each session consisted of three crossings. Testing was performed on postoperative days 12 and 28.

Head chamber implantation. After completing the behavioral training, an acrylic chamber was implanted on the skull under aseptic conditions for chronic mapping of the motor cortex. Each rat was anesthetized with ketamine hydrochloride $(70 \mathrm{mg} / \mathrm{kg})$ and xylazine $(10 \mathrm{mg} / \mathrm{kg})$. Dexamethasone $(0.1 \mathrm{mg} / \mathrm{kg}$; Banyu) was also administered intramuscularly to minimize the formation of brain edema. The head of the rat was fixed to the stereotaxic apparatus, the skull was widely exposed, and 8 small steel screws $(0.5 \mathrm{~mm}$ in diameter) were attached to the skull as anchors. The exposed skull and screws were cemented with dental acrylic and then a square acrylic chamber was mounted and fixed on the head of the rat. After $2 \mathrm{~d}$, the rat was set in the stereotaxic apparatus under anesthesia with ketamine/xylazine. A unilateral craniotomy was performed carefully to expose the primary motor cortex including the rostral and caudal 
forelimb areas on the ipsilesional side (Neafsey and Sievert, 1982; Rouiller et al., 1993). The dura remained intact and was kept moist with a $0.9 \%$ saline solution during surgery. After surgery, the exposed cortex was treated with gentamicin sulfate ointment (MSD) and the chamber was sealed tightly using the acrylic cover and screws. To prevent infection, the inside of the chamber was kept clean and the ointment was replaced every day.

ICMS. Intracortical microstimulation (ICMS) was performed at $5 \mathrm{~d}$ before and at 1,10 , and $26 \mathrm{~d}$ after ICH surgery. The animals were anesthetized with a mixture of ketamine $(70 \mathrm{mg} / \mathrm{kg})$ and xylazine $(10 \mathrm{mg} / \mathrm{kg})$ and additional doses of ketamine $(20 \mathrm{mg} / \mathrm{kg})$ were administered as necessary to maintain a stable level of anesthesia. Dexamethasone $(0.1 \mathrm{mg} /$ $\mathrm{kg}$ ) was given to reduce swelling of the cortex. The animals were stabilized in a stereotaxic frame. Glass insulated tapered tungsten electrodes $(0.5-1 \mathrm{M} \Omega$ impedance at $1 \mathrm{kHz}$, shaft diameter $310 \mu \mathrm{m}$; Alpha Omega Engineering) were used for stimulation. The electrode was lowered gently into the cortex until the tip reached cortical layer V (1400$1600 \mu \mathrm{m}$ below the cortical surface). Penetrations were made at $500 \mu \mathrm{m}$ intervals. The stimulation parameters were a $30 \mathrm{~ms}$ train of $200 \mu$ s duration biphasic pulses delivered at $333 \mathrm{~Hz}$ from an electrically isolated, constant current stimulator (STG4008; MultiChannelSystems). The stimulating current was increased gradually until joint and muscle movements could be detected. The minimum current required to elicit movements was recorded as the motor threshold. If no movements or twitches were evoked at $150 \mu \mathrm{A}$, the site was defined as "nonresponsive." The averaged threshold was also calculated and shown in Figure 2. After mapping, the gentamycin ointment was applied to the cortex and the chamber was sealed again and the animals were returned to their home cages for recovery.

Muscimol injection. To block neuronal activity, muscimol (SigmaAldrich), a $\mathrm{GABA}_{\mathrm{A}}$ receptor agonist, was injected into the rostral forelimb area (RFA) [3.5 mm anterior to the bregma (AP), $2.5 \mathrm{~mm}$ lateral to the bregma (ML) or caudal forelimb area (CFA) $(0.5 \mathrm{~mm} \mathrm{AP,} 3.0 \mathrm{~mm}$ $\mathrm{ML})$ ]. At $2 \mathrm{~d}$ after the completion of the second behavioral assessment ( $\mathrm{d}$ $26-28)$, the animals were anesthetized lightly using isoflurane $(\sim 1.0 \%)$ and set in the stereotaxic frame. First, $1 \mu \mathrm{l}$ of sterile saline $(0.9 \%)$ was injected into the center of the RFA or CFA, as determined by ICMS. At 30 min after injection, the rats were tested using the single-pellet reaching test and horizontal ladder test. Next, the rats were injected with the same amount of muscimol ( $1 \mu \mathrm{g} / \mu \mathrm{l}$ in $0.9 \%$ saline) into the RFA or CFA and behavioral performance was assessed. Each experiment was performed at an interval of $3 \mathrm{~d}$.

Anterograde tracing. At $39 \mathrm{~d}$ after ICH, biotin dextran amine (BDA, MW 10000; Invitrogen) was injected into the motor cortex of the injured hemisphere. BDA was delivered via a pulled glass capillary attached to a microliter Hamilton syringe at a flow rate of $100 \mathrm{~nL} / \mathrm{min}$ controlled by an electrical pump (ESP-64; Eicom). After anesthesia with a mixture of ketamine $(70 \mathrm{mg} / \mathrm{kg}$ ) and xylazine ( $10 \mathrm{mg} / \mathrm{kg}), 0.5 \mu \mathrm{l}$ of BDA (5\% in 0.1 M PBS) was injected at 4 sites in the forelimb area $(2.5 \mathrm{~mm} \mathrm{AP}, 2.0 \mathrm{~mm}$ $\mathrm{ML} ; 2.5 \mathrm{~mm}$ AP, $3.0 \mathrm{~mm}$ ML; 0.5 AP, $2.5 \mathrm{ML} ; 0.5 \mathrm{AP}, 3.5 \mathrm{ML})$. At 3 weeks after the injections, the animals were perfused transcardially with $0.1 \mathrm{M}$ PBS and 4\% paraformaldehyde (PFA) under deep anesthesia with pentobarbital sodium $(>100 \mathrm{mg} / \mathrm{kg}$, i.p.). The brains and spinal cords were dissected and postfixed and cryoprotected in 10\%,20\%, and 30\% sucrose in $0.1 \mathrm{M}$ PBS for cryostat sectioning in $40-\mu \mathrm{m}$-thick sections. The slices were processed with the $\mathrm{ABC}$ reaction protocol with nickel enhancement according to the manufacturer's manual (VECTASTAIN Elite ABC; Vector Laboratories). In brief, after several rinses in PBS, the slices were processed with the blocking solution $\left(0.6 \% \mathrm{H}_{2} \mathrm{O}_{2}, 20 \%\right.$ dimethyl sulfoxide in methanol) for $30 \mathrm{~min}$. Next, the sections were rinsed in PBS and incubated in PBS with 2\% ABC Elite reagent and 0.4\% Triton X-100 for $2 \mathrm{~h}$. The slices were rinsed in PBS and $10 \mathrm{~mm}$ Tris-buffered saline, and incubated in a diaminobenzidine (DAB)-Ni reaction solution $(0.01 \%$ DAB, $1.0 \%$ nickel ammonium sulfate, $0.0003 \% \mathrm{H}_{2} \mathrm{O}_{2}$ in Tris-buffered saline) for $30 \mathrm{~min}$. The stained sections were counterstained by neutral red. The stained slices were observed and recorded using light microscopy (Axioplan2; Zeiss and BZ-9000; Keyence). In each animal, BDApositive bouton-like swellings (varicosities) in contact with red nucleus neurons were counted from four sections within the red nucleus at $400 \times$ magnification. To normalize tracer uptake efficacy and lesion size, the total number of BDA-positive fibers in the cerebral peduncle was counted from 3 adjacent sections rostral to the red nucleus $(4.5-5.0 \mathrm{~mm}$ posterior to the bregma). The results are expressed as the mean number of bouton-like swellings in the red nucleus divided by the mean number of counted fibers in the cerebral peduncle for each animal. To compare the extent of the lesion of corticospinal tract, BDA-labeled fibers were counted at the levels of the cerebral peduncle and brainstem pyramid. Camera lucida drawing of the BDA-positive axons and varicosities was performed for each section at $400 \times$ magnification and all the labeled fibers and boutons were counted.

Selective pathway blockade by double-viral vector infection. To block the cortico-rubral pathway selectively, we combined two viral vectors comprising the Tet-on system as described previously (Lois et al., 2002, Kinoshita et al., 2012) with some modifications. In brief, we used a neuron-specific highly efficient retrograde gene transfer lentivirus (NeuRet) vector (Kato et al., 2011; Sooksawate et al., 2013) instead of the HiRet vector and adeno-associated type 1 virus (AAV1)- cytomegalovirus (CMV)- reverse tetracycline transactivator (rtTAV16) or AAV1$\mathrm{Ca}^{2+} /$ calmodulin-dependent protein kinase II (CaMKII)-rtTAV16 in place of AAV2-CMV-rtTAV16 because of the specificity and efficiency of its infection of neurons. The design of the two viral vectors is shown in Figure $5 a$.

At $35 \mathrm{~d}$ after ICH, the animals were anesthetized with a ketamine (70 $\mathrm{mg} / \mathrm{kg})$ and xylazine $(10 \mathrm{mg} / \mathrm{kg})$ mixture and injected with atropine $(0.1$ $\mathrm{mg} / \mathrm{kg})$ and dexamethasone $(0.1 \mathrm{mg} / \mathrm{kg})$ for premedication. The rats were fixed to the stereotaxic apparatus and a small hole was drilled in the skull above the red nucleus. Two injections of NeuRet-tetracyclineresponsive element (TRE)-enhanced green fluorescent protein (EGFP)enhanced tetanus neurotoxin light chain (eTeNT) $(0.4 \mu \mathrm{l}$ each; titer, $2.2-2.6 \times 10^{11}$ copies $/ \mathrm{ml}$ ) were made using pulled glass micropipette connected to a microsyringe and syringe pump ( $-5.3 \mathrm{~mm} \mathrm{AP}, 1.7 \mathrm{~mm}$ $\mathrm{ML}, 8.6 \mathrm{~mm}$ below the skull surface; $-5.8 \mathrm{~mm}$ AP, $1.7 \mathrm{~mm} \mathrm{ML}, 8.3 \mathrm{~mm}$ depth). The micropipette was lowered at an angle of $5^{\circ}$ laterally deviated from the vertical axis to avoid damage to the sinus and subsequent hemorrhage. Injection was performed at a rate of $0.1 \mu \mathrm{l} / \mathrm{min}$ over $4 \mathrm{~min}$ and the needle was left in place for 4 min to prevent reflux and then removed slowly. At 10-14 d after NeuRet-TRE-EGFP.eTeNT injection, a craniotomy was performed, exposing the ipsilesional motor cortex forelimb region. AAV1-CMV-rtTAV16 $\left(0.4 \mu \mathrm{l}\right.$; titer, $\left.8.0 \times 10^{10} \mathrm{vg} / \mu \mathrm{l}\right)$ or AAV1CaMKII-rtTAV16 $\left(0.4 \mu \mathrm{l}\right.$; titer, $\left.2.2 \times 10^{10} \mathrm{vg} / \mu \mathrm{l}\right)$ was injected into the primary motor cortex at 8 sites $(1.0 \mathrm{~mm} \mathrm{AP,} 2.5 \mathrm{~mm} \mathrm{ML} ; 1.0 \mathrm{~mm} \mathrm{AP}, 3.5$ $\mathrm{mm} \mathrm{ML} ; 1.5 \mathrm{~mm}$ AP, $2.0 \mathrm{~mm} \mathrm{ML} ; 2.0 \mathrm{~mm}$ AP, $2.5 \mathrm{~mm}$; AP, $2.0 \mathrm{~mm}$, ML, $3.5 \mathrm{~mm}$; AP, $2.5 \mathrm{~mm}$, ML, $3.0 \mathrm{~mm}$; AP, $3.0 \mathrm{~mm}$, ML, $2.5 \mathrm{~mm}$; AP, $3.0 \mathrm{~mm}$ $\mathrm{AP}, 3.5 \mathrm{~mm} \mathrm{ML}$ ). Each injection was performed at $1.5 \mathrm{~mm}$ below the skull surface for $4 \mathrm{~min}$ at a rate of $0.1 \mu \mathrm{l} / \mathrm{min}$.

At 6 weeks after AAV injection, oral administration of doxycycline (DOX) in the drinking water was initiated $(3 \mathrm{mg} / \mathrm{ml}$ in a $5 \%$ sucrose solution). DOX administration was continued for 3 weeks. In addition, a single intraperitoneal injection of DOX (10 $\mu \mathrm{g} / \mathrm{g}$ body weight, in $0.9 \%$ $\mathrm{NaCl}$ ) was given on the first day of oral DOX administration. In parallel with DOX administration, behavioral testing (skilled reaching and horizontal ladder stepping) was conducted. The animals were tested at $1 \mathrm{~d}$ before the initiation of DOX administration (pre-DOX, post-ICH day 91) and followed by daily testing for first week of administration (postDOX, post-ICH day 92-98) and weekly for next 2 weeks. Skilled reaching trials were videotaped from both side and front and the trajectories of reaching movements were processed in further detailed analyses. The distance from the slit located in the front wall of the testing apparatus to the farthest point of each reaching trajectory was measured using frameby-frame analysis (60 frames/s). Averaged reaching distance was calculated from each testing session. In addition, the range of wrist rotation angle throughout reaching was measured from front view video analysis (200 frames/s). Wrist pronation angle was calculated when the rat intended to grasp a pellet and supination angle was measured from the withdrawal phase of reaching movement after grasping. Averaged range of motion was calculated from each testing session.

After the last assessment, the animals were perfused transcardially with $4 \%$ PFA under deep anesthesia by sodium pentobarbital $(>80 \mathrm{mg} / \mathrm{kg})$ 
and the brain was dissected and postfixed. After cryoprotection, $40 \mu \mathrm{m}$ coronal sections were made using a sliding microtome (HM450; Microm). Some sections were processed for anti-EGFP immunohistochemistry to confirm the presence of double-infected neurons. In addition, the distribution of neurons infected by AAV was confirmed by in situ hybridization to detect the rtTA sequence (the antisense probe for the tetracycline transactivator, tTA, was a gift from $A$. Watakabe, National Institute for Basic Biology, Okazaki, Japan). It was confirmed that the AAV vector had infected a majority of the neurons in the ipsilesional motor cortex forelimb area. The technical details of anti-EGFP immunohistochemistry and in situ hybridization against the rtTA sequence were described previously (Kinoshita et al., 2012) and are available online (http://www.nibb.ac. $\mathrm{jp} / \mathrm{brish} / \mathrm{indexE}$.html). Photographs of the histological slices were taken using a light microscope (Axioplan2 and BZ-9000).

Statistical analysis. Statistical analysis was conducted using JMP version 10 (JMP statistical software; SAS Institute). A significance level of $p<0.05$ was chosen. Behavioral assessments were analyzed by parametric ANOVA, followed by the post hoc Tukey-Kramer's post hoc test. Dunnett's test was used for comparing the performance of forelimb movements before and during DOX administration. The number of BDA-positive varicosities in the red nucleus was analyzed using Student's $t$ test. Data are presented as means and SEM for Figures 1, 3, and 4 and as means and SD for Figures 5 and 6 .

\section{Results}

FLU improves recovery of impaired forelimb function after capsular hemorrhage

To clarify the causal relationship between the reorganization of the injured neural circuits and functional recovery, we used the same Wistar rats for longitudinal behavioral, electrophysiological, and morphological analyses. Figure $1 a$ indicates the experimental time course of the study. We made a capsular hemorrhage model by collagenase injection into the unilateral IC. To quantify the lesion extent of IC between the two groups of animals, we performed Klüver-Barrera staining at the level of the IC on both sides and calculated the lesion extent after binarization of images (Fig. 1c,d) normalized to the IC on the intact side. As a result, the percentage of the damaged corticofugal fibers in the IC was not significantly different between groups (ICH: $61.5 \pm 6.9 \%$; ICH-FLU: $56.1 \pm$ $10.2 \%$; unpaired $t$ test: $p=0.699)$. We also counted the number of BDA-labeled corticofugal fibers in the cerebral peduncle and the brainstem pyramid. There was no significant difference between the five ICH and seven ICH-FLU rats (cerebral peduncle: ICH; $270.6 \pm 117.0$, ICH-FLU; $325.7 \pm 59.6$, unpaired $t$ test, $p=$ $0.721, t$ test, brainstem pyramid: ICH; $180 \pm 82.5$, ICH-FLU; $236.1 \pm 42.9$, unpaired $t$ test, $p=0.721$ ). The majority of CST fibers running through the IC were injured in this model

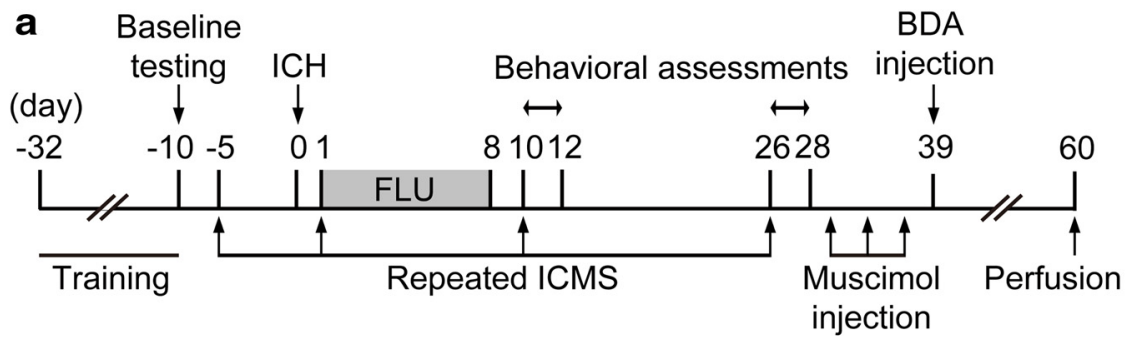

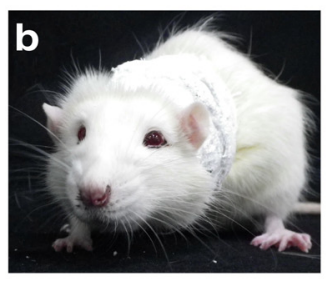

e

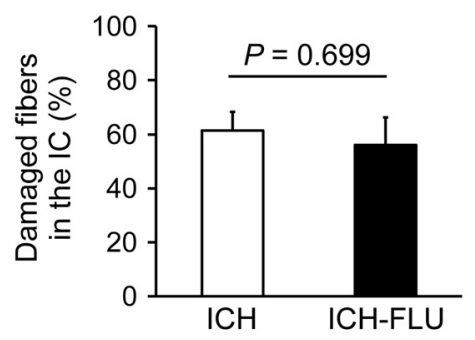

g

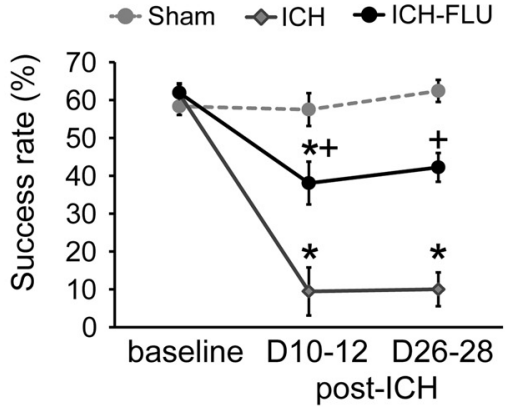

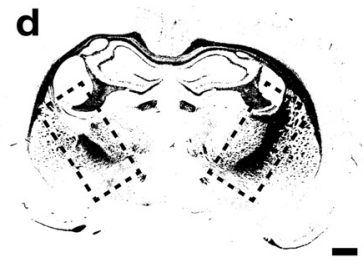

f
Figure 1. Experimental setup and behavioral effects of forced limb use. $\boldsymbol{a}$, Diagram showing the timeline of the experiments. $\boldsymbol{b}$, Application of FLU treatment. A one-sleeve cast was fitted the rat to restrain its unimpaired limb and force the animal to use its impaired limb. c, Representative Klüver-Barrera-stained image of an ICH. The arrow indicates the hemorrhage site induced by collagenase injection. Scale bar, $1 \mathrm{~mm}$. $\boldsymbol{d}$, Binarized image of $\boldsymbol{c}$. Dashed rectangles indicate the IC. $\boldsymbol{e}$, Percentage of the damaged fibers in the IC. There was no significant difference between groups $(p=0.699)$. $f$, Motor deficits score. Comparable impairment of gross motor functions between ICH group and ICH-FLU group were shown. $\boldsymbol{g}$, Single-pellet reaching test. The ICH-FLU group showed a significantly better performance than the ICH control group on days $10-12$ and $26-28$ after ICH. ${ }^{*} p<0.05$ vs sham; $+p<0.05$ vs ICH. $\boldsymbol{h}$, Horizontal ladder stepping test. ICH-FLU rats demonstrated a lower fall rate than ICH rats. ${ }^{*} p<0.05$ vs sham, $+p<0.05$ vs ICH. Values are shown as mean \pm SEM.

(Masuda et al., 2007; Ishida et al., 2011), resulting in severe impairment of gross motor function (Fig. $1 f$ ). Based on these findings, the lesion extent was considered not to be significantly different between the ICH and ICH-FLU groups. Some ICH rats were fitted with a one-sleeve plaster cast so they were forced to use their impaired limb for all their living activities on postoperative days 1-8 (Fig. 1b). For all three groups of rats (sham, nontreated $\mathrm{ICH}$, and ICH-FLU), performance of the reaching test was almost the same at the baseline assessment (Fig. 1e). The performance of skilled reaching test and horizontal ladder test showed little change in sham-operated rats [baseline: $58.3 \pm 2.3 \%$; post- 
a

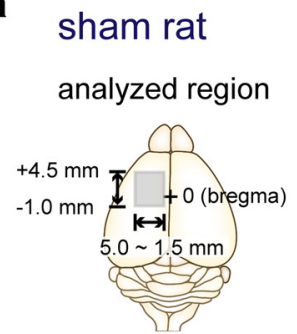

b

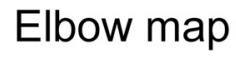

$\mathrm{ICH}$ group

ICH-FLU

group

c Wrist map

$\mathrm{ICH}$ group

ICH-FLU

group

\section{d Averaged} Threshold

\section{pre-ICH}
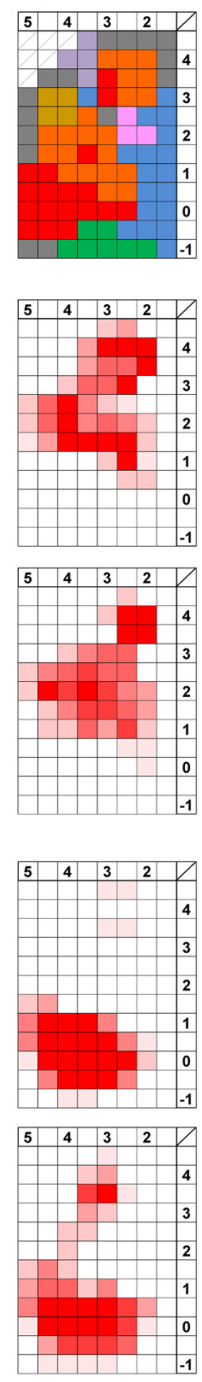
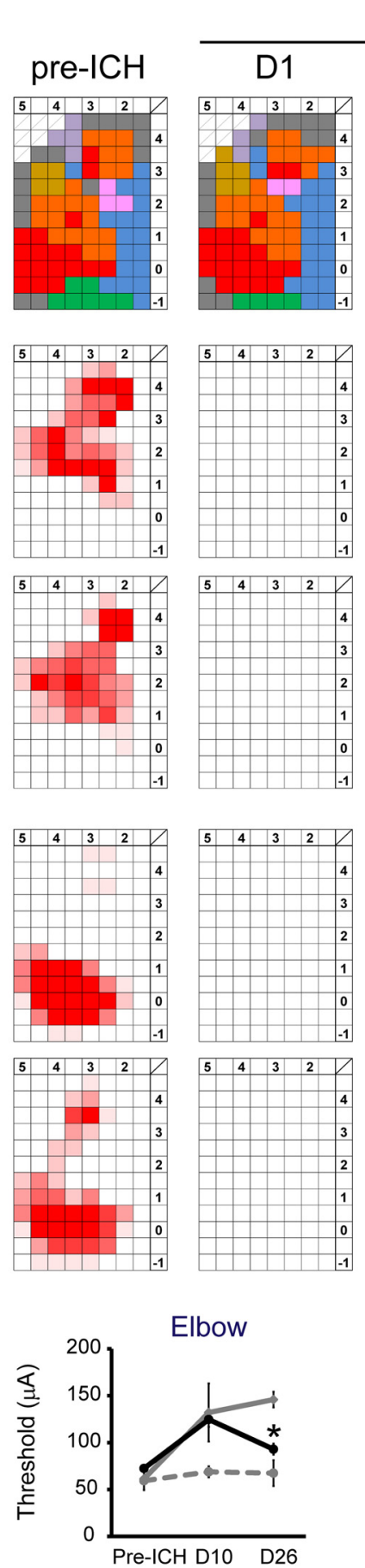

\section{post-ICH}

D10 D26
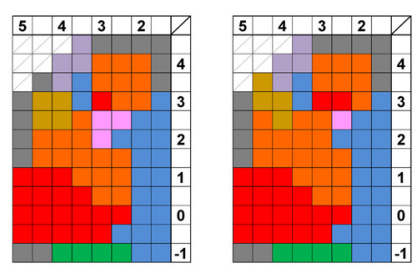

$\square$ elbow

$\square$ wrist

$\square$ vibrissa

$\square$ hip

$\square$ neck

$\square$ face

$\square$ jaw

$\square$ no responce
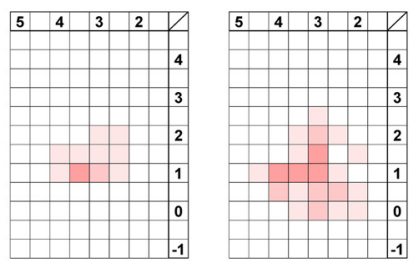

the number of the

responsive animals
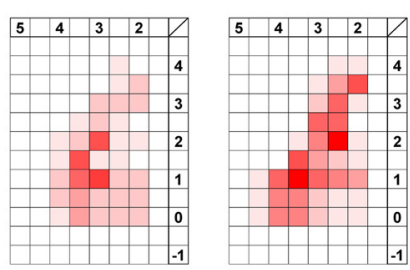

$n=6$

$n=5$

$\mathrm{n}=4$

$n=3$

$\mathrm{n}=2$

$n=1$
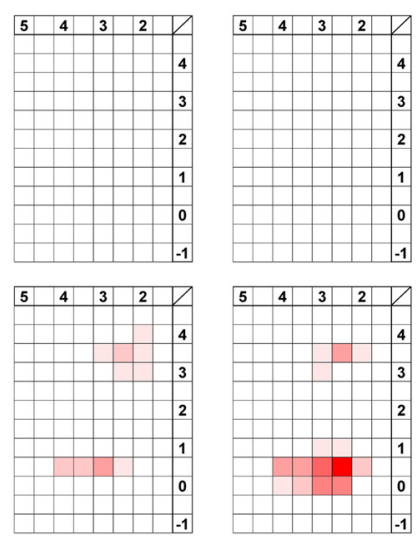

Wrist

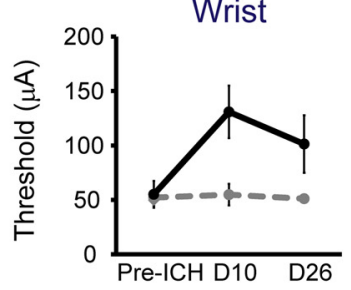

- Sham

$+\mathrm{ICH}$

- ICH-FLU

Figure 2. Temporal changes of the forelimb representation map in the ipsilesional motor cortex. $\boldsymbol{a}$, Typical motor representative map of the motor cortex of a sham rat. ICMS was conducted to make the representative map at $10 \mathrm{~d}$ before $\mathrm{ICH}$ and at 1, 10, and $26 \mathrm{~d}$ after ICH. The analyzed region is indicated with the gray rectangle in the left panel (AP: from $4.5 \mathrm{~mm}$ anterior to the bregma to $1.0 \mathrm{~mm}$ posterior to the bregma; $\mathrm{ML}$ : from $5.0 \mathrm{~mm}$ to $1.5 \mathrm{~mm}$ lateral to the bregma). Penetrations and stimulations were performed at $500 \mu \mathrm{m}$ intervals. Each color represents the body part in which movements were induced at the lowest stimulus strength. $\boldsymbol{b}$, Elbow representation map on the forelimb motor cortex. The upper four panels indicate the elbow map of the ICH group and the lower four panels indicate the map of the ICH-FLU group. Density of red shows the number of responding animals per cell (maximally $n=6$ ). c, Wrist representation map. $\boldsymbol{d}$, Averaged threshold of the elbow and wrist map. Elbow threshold of the ICH-FLU group was lower than that of the ICH group at $26 \mathrm{~d}$ after ICH. ${ }^{*} p<0.05$ vs ICH. Values are shown as mean \pm SEM.

operative days 10-12 (D10-12): $57.5 \pm 4.3 \%$; D26-28: $62.4 \pm$ 2.9\%; and baseline: $3.9 \pm 0.7 \%$; D10-12: $3.4 \pm 0.8 \%$; D26-28: $3.9 \pm 0.7 \%$, respectively). After $7 \mathrm{~d}$ of FLU treatment, performance in the skilled reaching test was improved in $\mathrm{ICH}$ rats with FLU treatment compared with the ICH group on D10-12 (ICH: $9.4 \pm 6.3 \%$; ICH-FLU: $38.1 \pm 5.7 \%$; Tukey-Kramer's test: $p=$ 0.026; Fig. $1 g$ ) and D26-28 (ICH: $10.0 \pm 4.5 \%$; ICH-FLU: $42.2 \pm$
3.8\%; Tukey-Kramer's test: $p=0.006)$. Similarly, the ICH-FLU group showed a significantly lower fall rate than the ICH group in the horizontal ladder test on days 12 (ICH: $23.2 \pm 3.6 \%$; ICHFLU: $9.9 \pm 1.6 \%$; Tukey-Kramer's test: $p=0.006$; Fig. $1 \mathrm{~h}$ ) and 28 (ICH: $18.8 \pm 2.1 \%$; ICH-FLU: $7.6 \pm 1.7 \%$; Tukey-Kramer's test: $p=0.001)$. The performance of the ICH-FLU group recovered to almost the same level as the sham animals at day 28. These results 
were consistent with our previous report (Ishida et al., 2011), and indicated that FLU improved skilled forelimb motor function after ICH.

\section{FLU promotes reorganization of the output effects from the ipsilesional motor cortex}

To reveal FLU-induced reorganization of the injured neural circuits, we analyzed the changes of the forelimb representative area of the ipsilesional motor cortex by ICMS in the same rats used for the behavioral assessments throughout the recovery course (Fig. 1a). Temporal changes in cortical mapping are shown in Figure 2. Two major areas with forelimb representation were revealed by ICMS in the frontal cortex (AP: 4.0-3.0, ML: 2.0-3.0; AP: 2.00.0, ML: 2.5-4.5), which have been reported as the RFA and CFA, respectively. Repetition of ICMS itself did not appear to induce visible changes in the cortical map (Fig. 2a); there was no clear difference among the groups before ICH surgery (Fig. $2 b, c)$. One day after $\mathrm{ICH}$, the elbow and wrist representative areas disappeared totally in both the ICH and ICH-FLU groups. Probably due to poststroke spontaneous recovery, 3 of $6 \mathrm{ICH}$ rats showed reemergence of the elbow area at $10 \mathrm{~d}$ after $\mathrm{ICH}$, which was detected in all the ICH rats at day 26. However, this "reemerged elbow area" was much smaller than the pre-ICH elbow area and was shifted to the mediocaudal part of the CFA (Fig. $2 b$ ). No wrist area was found in the $\mathrm{ICH}$ rats after $\mathrm{ICH}$ (Fig. $2 c$ ). In addition, the averaged motor threshold of elbow movements was greatly elevated at day 10 and 26 compared with the pre-ICH period (Fig. $2 d$ ). Conversely, 6 of $7 \mathrm{ICH}-\mathrm{FLU}$ rats showed the reappearance of the elbow and wrist areas at days 10 and all the ICH-FLU rats did at day 26 (Fig. 2b,c). This reemerged area in the ICH-FLU group was also smaller than in the pre-ICH rats; however, ICH-FLU rats exhibited larger elbow and wrist representative areas at days 10 and 26 compared with the ICH group (Fig. $2 b, c$ ). In addition, these areas were distributed, not only in the CFA, but also in the RFA (Fig. $2 b, c$ ). The averaged motor-evoked threshold of elbow movements was significantly lower in the ICH-FLU group than in the ICH group at day 26 (Tukey-Kramer's test: $p=0.036$, Fig. $2 d$ ). The motorevoked threshold of wrist movements also showed a tendency to decrease in the post-ICH phase. These results indicated that FLU promoted reorganization of the ipsilesional forelimb area and reorganization of the descending connections from those areas to spinal motoneurons was suggested.

\section{Reemerged rostral and caudal forelimb regions are involved in functional recovery induced by FLU}

To investigate the possible role of the reemerged forelimb representative areas in the ICH-FLU animals, the areas were inactivated by local injections of the GABA type $A\left(G A B A_{A}\right)$ receptor agonist muscimol. Microinjections of muscimol or saline into the RFA or CFA were followed by behavioral assessments at $30 \mathrm{~min}$ after injection. First, as expected, the ICH-FLU group showed better performance than those in the ICH group after saline injection in the skilled reaching task $(\mathrm{ICH}: 15.0 \pm 5.8 \%$; ICH-FLU: $38.3 \pm 4.4 \%$; unpaired $t$ test: $p=0.033$; Fig. $3 a$ ). After muscimol injection into the RFA or CFA, ICH-FLU rats showed a significant decrease of reaching success rate compared with the rate b

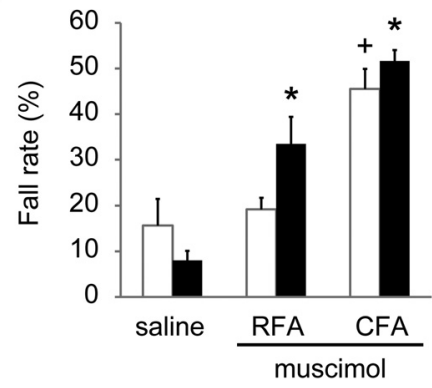

after saline injection (RFA: $3.3 \pm 1.7 \%$; paired $t$ test: $p=0.01$ CFA: $3.3 \pm 3.3 \%$; paired $t$ test: $p=0.022$; Fig. $3 a$ ). The rats showed great difficulty in reaching to pellet through a narrow slit, indicating that their reaching skill was severely impaired after the muscimol injection. Although the ICH group also showed a significant deterioration of reaching success rate after muscimol injection into the CFA $(1.7 \pm 1.7 \%$; paired $t$ test: $p=0.047)$, their performance did not alter significantly after RFA injection (12.5 $\pm 4.3 \%$; paired $t$ test: $p=0.024)$. Similarly, the ICH-FLU group demonstrated a significant increase of fall rate during horizontal ladder stepping after muscimol injection into the RFA or CFA compared with saline injection (saline: $8.0 \pm 2.1 \%$; RFA: $33.5 \pm 5.9 \%$, paired $t$ test: $p=0.01$; CFA: $51.7 \pm 2.4 \%$, paired $t$ test: $p=0.022$; Fig. $3 b$ ) The ICH group also showed a significant worsening of stepping movements after muscimol injection into the CFA compared with saline injection (saline: $15.7 \pm 5.8 \%$; CFA: $45.5 \pm$ $4.4 \%$; paired $t$ test: $p=0.014)$, but this was not the case for RFA injection (RFA: $19.2 \pm 2.5 \%$; paired $t$ test: $p=0.626$ ). These data, consistent with the results of ICMS mapping, suggest that the reemerged forelimb representative map of CFA and RFA contributed to regaining the impaired forelimb functions and, in particular, RFA was primarily involved in the FLU-induced functional recovery.

\section{Substantial descending projections from the ipsilesional motor cortex after FLU}

Next, we conducted anterograde tracing of axonal projections from the reemerged forelimb area of the ipsilesional motor cortex to clarify the targets of these descending projections. The anterograde tracer BDA was injected into the ipsilesional motor cortex forelimb area in the rats used in the assessments for behavior and motor representation (see Fig. 1a).

BDA was injected into four sites at $500 \mu \mathrm{m}$ intervals in the ipsilesional cortex forelimb area spanning the RFA and CFA of both ICH (Fig. $4 a, a^{\prime}$ ) and ICH-FLU rats (Fig. $4 e, e^{\prime}$ ). The size and location of the lesion caused by the collagenase-induced hemorrhage around the IC were similar in both the ICH $(n=5)$ and ICH-FLU $(n=7)$ groups (Figs. $\left.1 c-e, 4 b, b^{\prime}, f, f^{\prime}\right)$. The lesions located within the IC appeared to have injured the majority of corticofugal fibers; however, a small number of BDA-labeled fibers remained around the lesion (Fig. $4 b^{\prime}, f^{\prime}$ ). The extent of lesion was found to be comparable between the ICH and ICH-FLU 


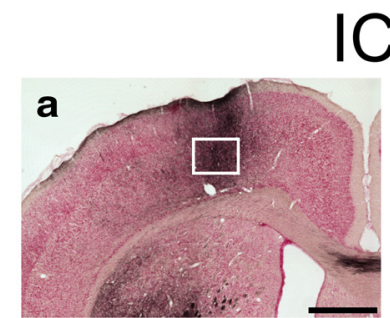

$\mathrm{ICH}$
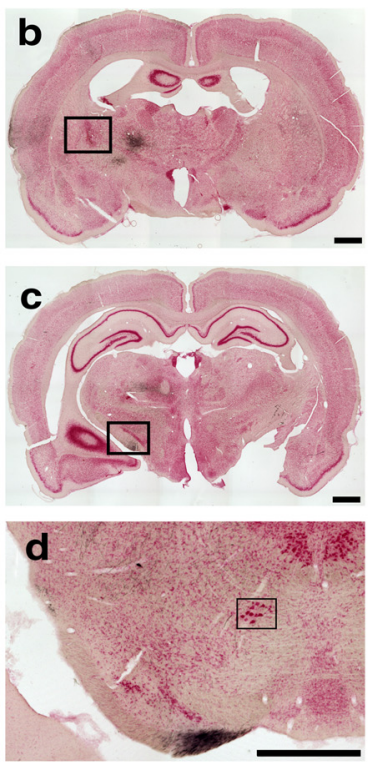

i
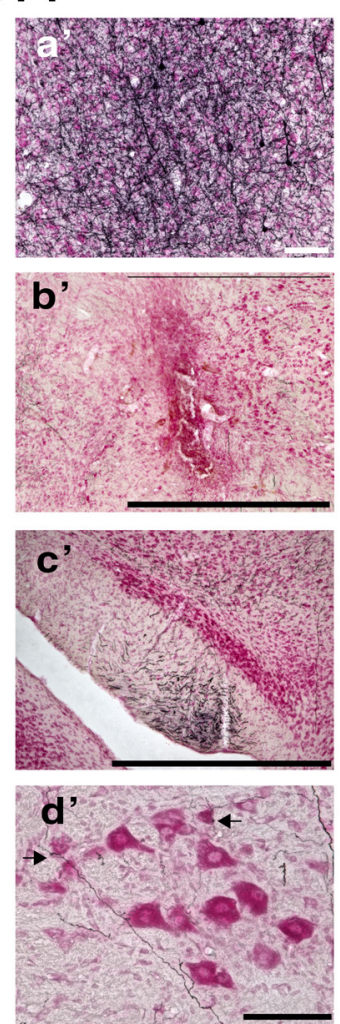

j
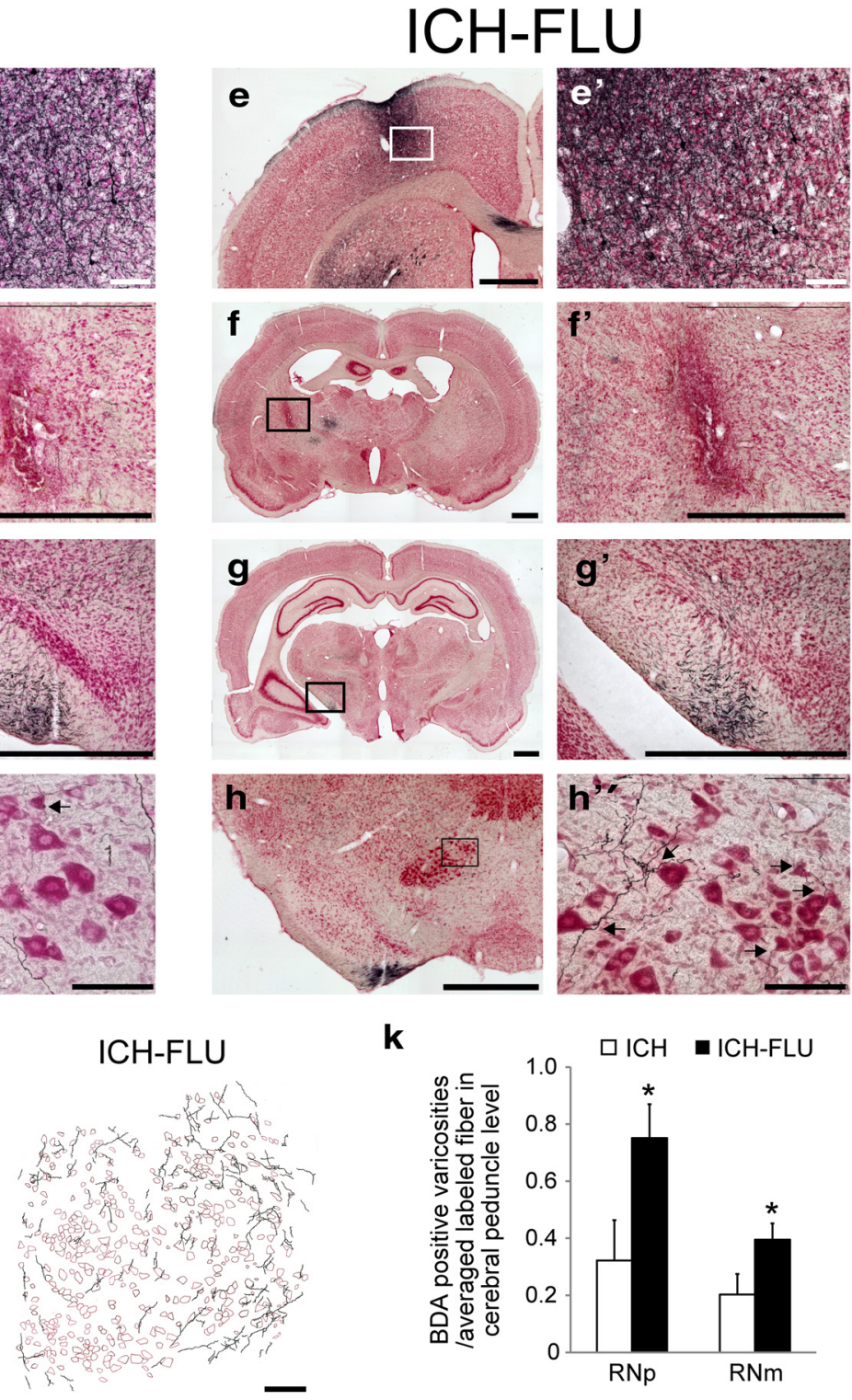

k

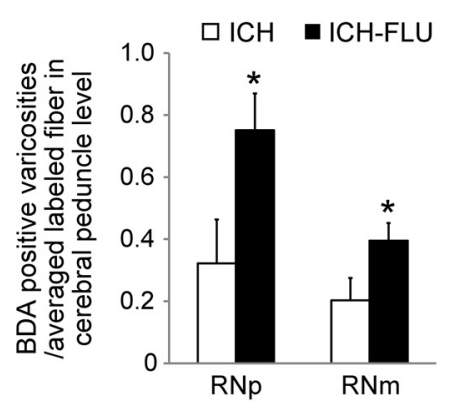

Figure 4. Axonal projections from the ipsilesional forelimb motor cortex after ICH and ICH-FLU. To characterize the anatomical reorganization of the ipsilesional forelimb area, anterograde tracing assay using BDA was conducted. $\boldsymbol{a}^{\prime}-\boldsymbol{h}^{\prime}$ show a higher magnification of $\boldsymbol{a}-\boldsymbol{h} . \boldsymbol{a}, \boldsymbol{e}$, Injection site of BDA in the ipsilesional motor cortex forelimb area. $\boldsymbol{b}, \boldsymbol{f}$, ICH lesion site. Most of the lesions caused by ICH were located in the IC and some intact corticofugal fibers still remained around the lesion. $\boldsymbol{c}, \boldsymbol{g}$, BDA-positive fibers running through the cerebral peduncle. $\boldsymbol{d}, \boldsymbol{h}$, Red nucleus (magnocellular part). Compared with the ICH group $\left(\boldsymbol{d}^{\prime}\right)$, many BDA-labeled fibers were observed around the ipsilesional red nucleus in the ICH-FLU group $\left(\boldsymbol{h}^{\prime}\right)$. Arrows indicate boutons along axons contacting large-sized red nucleus neurons. $\boldsymbol{i}, \boldsymbol{j}$, Camera lucida drawing of the BDA-positive axons in the red nucleus. $\boldsymbol{k}$, Quantitative analysis for the boutons of the cortico-rubral axons. The number of BDA-positive boutons in the RNp and RNm normalized by the total number of labeled axons was significantly higher in the ICH-FLU group than in the ICH group. Scale bar, $1000 \mu \mathrm{m}$ for $\boldsymbol{a}-\boldsymbol{h}, \boldsymbol{c}^{\prime}$, and $\boldsymbol{g}^{\prime} ; 500 \mu \mathrm{m}$ for $\boldsymbol{b}^{\prime}$ and $\boldsymbol{f}^{\prime} ; 100 \mu \mathrm{m}$ for $\boldsymbol{a}^{\prime}, \boldsymbol{d}^{\prime}, \boldsymbol{e}^{\prime}, \boldsymbol{h}^{\prime}, \boldsymbol{i}$ and $\boldsymbol{j}$. ${ }^{*} p<0.05 \mathrm{vs}$ ICH. Values are shown as mean \pm SEM.

groups (see above). Interestingly, a larger number of BDApositive fibers were found in and around the ipsilesional red nucleus in the ICH-FLU group (Fig. 4h). BDA-positive axons were distributed in both the parvocellular and magnocellular part of the red nucleus (RNp and RNm, respectively) and varicosities of these fibers were found adjacent to both RNp and RNm neurons (Fig. $4 h^{\prime}, j$ ). Although a few BDA-positive fibers were found in the red nucleus also in the ICH rats (Fig. $4 d, d^{\prime}, i$ ), the number of BDA-positive varicosities was $97.4 \pm 59.9$ in $\mathrm{RNp}$ and $35.6 \pm$ 18.8 in RNm of ICH rats and $273.4 \pm 37.6$ in RNp and $154.2 \pm$ 17.9 in RNm of ICH-FLU rats. Therefore, the number of varicosities per labeled fiber through cerebral peduncle (see above) significantly increased in ICH-FLU group compared with ICH group both in the RNp (ICH: $0.32 \pm 0.14$, ICH-FLU: $0.75 \pm 0.12$, unpaired $t$ test: $p=0.043$, Fig. $4 k$ ) and RNm (ICH: $0.20 \pm 0.07$, ICH-FLU: $0.42 \pm 0.07$, unpaired $t$ test: $p=0.042$ ). These results suggested that the ipsilesional motor cortex, which showed reorganization and expansion after ICH and intensive use of the impaired forelimb, had substantial axonal projections to both the $\mathrm{RNp}$ and $\mathrm{RNm}$.

\section{Ipsilesional cortico-rubral pathway is essential for recovery induced by FLU}

The above results suggested that the recovery of forelimb function after ICH in the ICH-FLU group was caused by reconstruction of the cortico-rubro-spinal descending pathway. We tested this hypothesis by using selective and reversible inactivation of cortico-rubral pathway (Kinoshita et al., 2012; Sooksawate et al., 
a

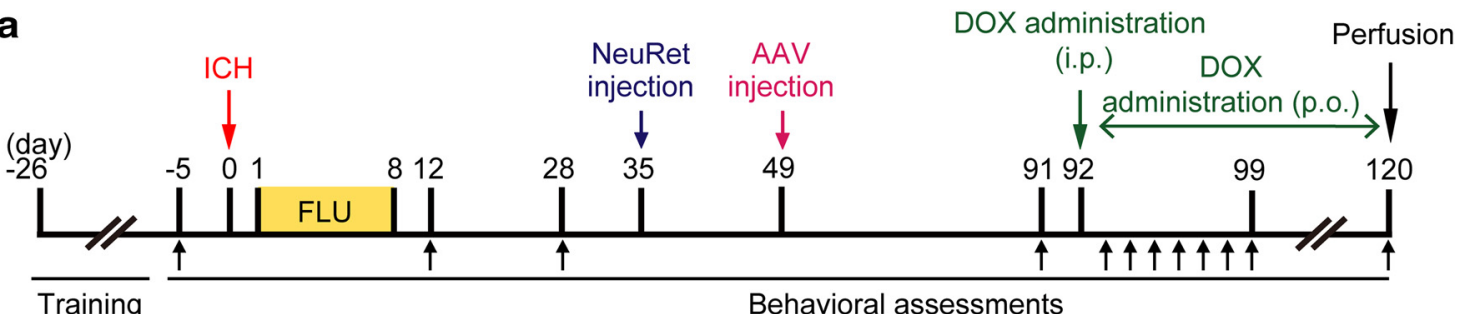

Training

Behavioral assessments

b

NeuRet-TRE-EGFP.eTeNT
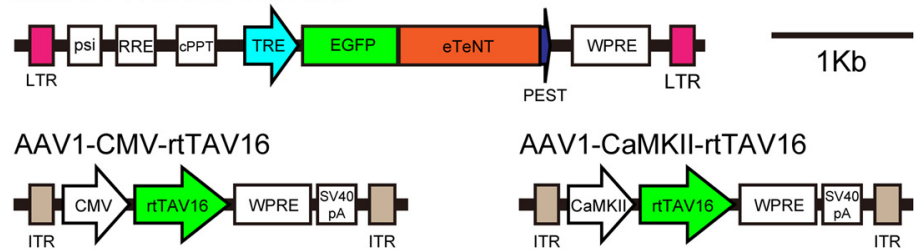

AAV1-CaMKII-rtTAV16

reaching
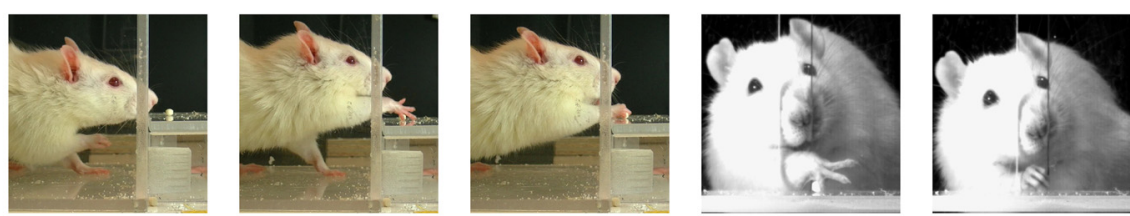

post-DOX
reaching (D2)
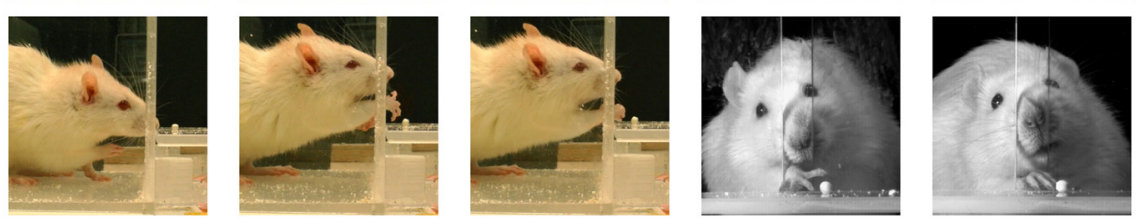

d

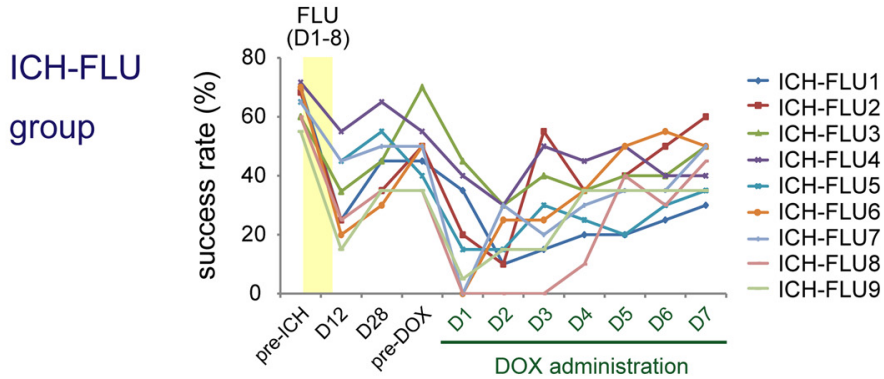

$\mathrm{ICH}$ group

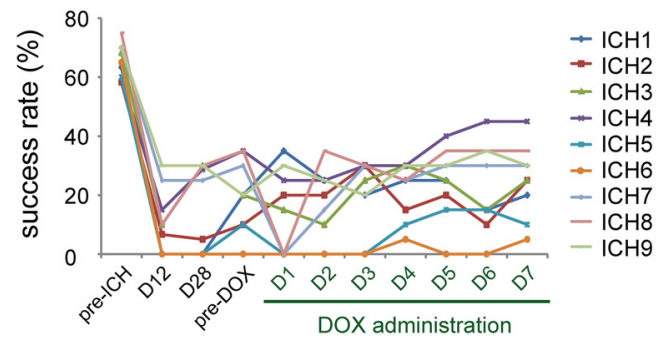

e

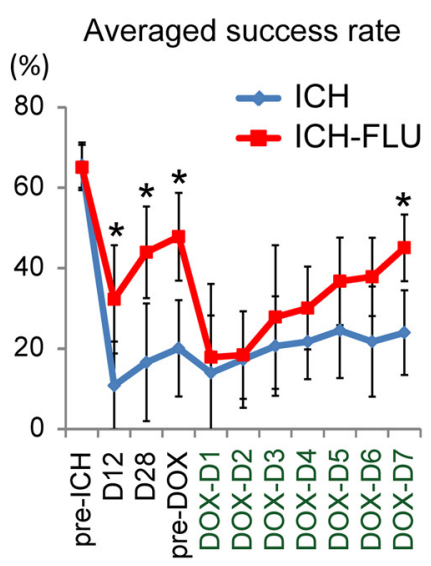

Figure 5. Effects of selective blockade of the cortico-rubral pathway on reaching function. To investigate the causal relationship between the cortico-rubral projections and functional recovery in ICH-FLU rats, selective blockade was performed using a double-infection technique. (a) Time course of the double-infection experiment. After ICH and FLU treatment, lentivirus and AAV were injected into the red nucleus and motor cortex, respectively. $\boldsymbol{b}$, Design of the viruses. Synaptic transmissions of double-infected neurons with NeuRet and AAV1 vectors were blocked in response to DOX administration. $\boldsymbol{c}$, Representative images of the reaching movement of an ICH-FLU rat before and after DOX administration. After DOX administration, the rat showed impaired reaching movements. $\boldsymbol{d}$, Serial measurements of reaching function for each individual ICH-FLU and ICH rat. Each line indicates the change of the reaching success rate of each rat. Top and bottom panels demonstrate the results of the ICH-FLU and ICH groups, respectively. For ICH-FLU rats, recovery of reaching function was observed until $1 \mathrm{~d}$ before DOX administration. After DOX administration, their performance dropped drastically. In contrast, ICH rats did not show a major change of performance, except for a few rats. $\boldsymbol{e}$, Average of the reaching success rate of both groups. Performance of the reaching test by the ICH-FLU group fell to the same level as the ICH group in response to DOX administration. At day 7, the success rate of the ICH-FLU group recovered gradually. ${ }^{*} p<0.05$ vs ICH. Values are shown as mean \pm SD.

2013). Figure $5 a$ indicates the experimental design and time course. We injected neuron-specific highly efficient retrograde gene transfer lentiviral (NeuRet) vectors expressing the fusion gene of eTeNT (Yamamoto et al., 2003) and EGFP under control of the TRE into the ipsilesional red nucleus, and then we injected AAV1 vectors expressing the rtTAV16 gene under control of the CMV or the CaMKII promotor into the motor cortex ipsilateral to the ICH (Fig. 5b). Therefore, cortical neurons with axons that 


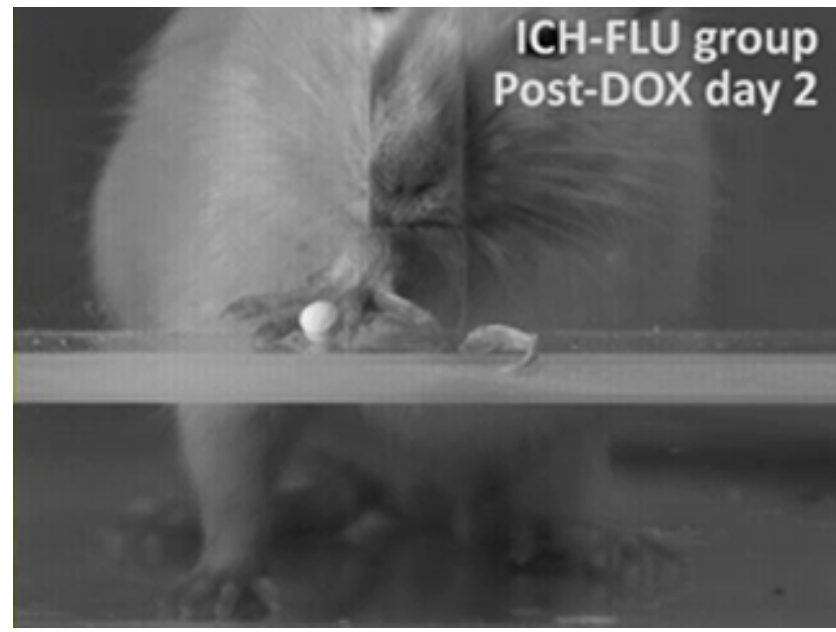

Movie 1. Examples of reaching movement of ICH-FLU group rat on $5 \mathrm{~d}$ before $\mathrm{ICH}$ and $1 \mathrm{~d}$ before and $2 \mathrm{~d}$ after the initiation of DOX administration from a frontal view. Selective blockade of the cortico-rubral pathway abolished the FLU-induced recovery of reaching function.

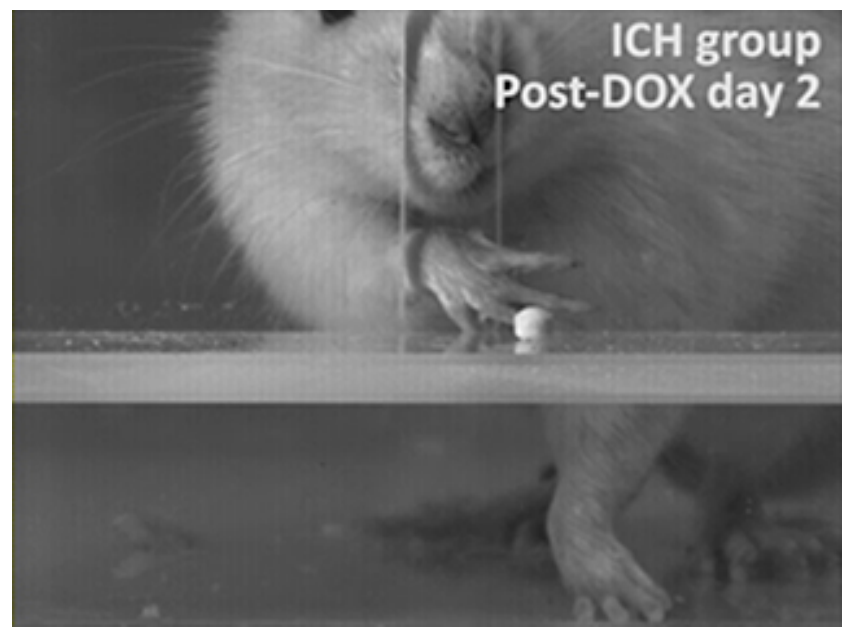

Movie 2. Examples of reaching movement of $\mathrm{ICH}$ group rat on $5 \mathrm{~d}$ before $\mathrm{ICH}$ and $1 \mathrm{~d}$ before and $2 \mathrm{~d}$ after the initiation of DOX administration from a frontal view. Nontreated control rat did not show apparent recovery of reaching function after $\mathrm{ICH}$.

project to the red nucleus were expected to be double infected. In a previous study (Kato et al., 2011), the retrograde labeling with the NeuRet lentiviral vector was found only between the structures that were known to be connected with each other and there was no sign of uptake by the passing fibers. Therefore, the possibility of infection of the corticospinal neurons was discarded. In fact, we could not find any GFP-positive fibers in the cerebral peduncle caudal to the red nucleus in the ICH-FLU rats. The double-infected cortico-rubral neurons express eTeNT, which depresses synaptic transmission during DOX administration.

As shown in Figure $1 a$, ICH-FLU rats showed a higher success rate in the single-pellet reaching test than control rats at days 12 and 28 after ICH (Fig. $5 d$ ). In addition, the ICH-FLU group also demonstrated better performance (47.8 $\pm 10.9 \%)$ compared with the ICH group $(20.0 \pm 12.0 \%)$ on the day before DOX administration (pre-DOX, at D91; unpaired $t$ test: $p<0.001$; Fig. $5 e)$. ICH-FLU rats precisely guided their impaired forelimb to the pellet and grasped it tightly with their digits (Fig. $5 c$ ). After DOX administration, the reaching success rate of the ICH-FLU group dropped to the same level as that of the ICH group (D2: $18.3 \pm$

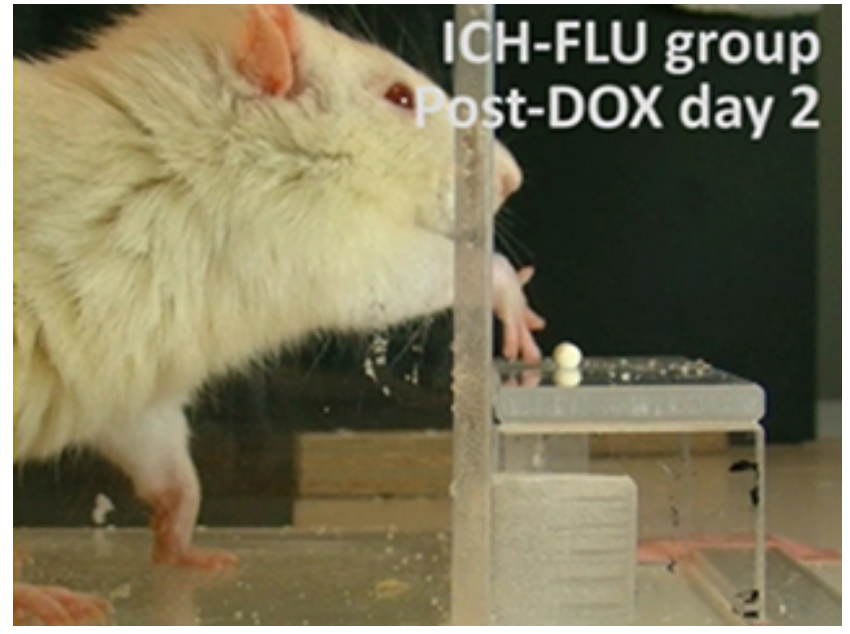

Movie 3. Examples of reaching movement of ICH-FLU group rat on $5 \mathrm{~d}$ before $\mathrm{ICH}$ and $1 \mathrm{~d}$ before and $2 \mathrm{~d}$ after the initiation of DOX administration from a lateral view.

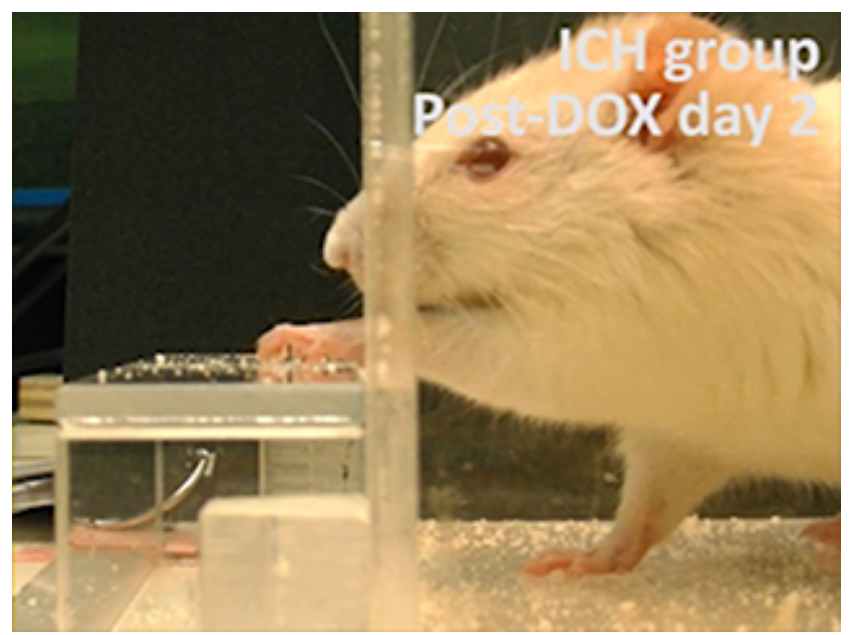

Movie 4. Examples of reaching movement of $\mathrm{ICH}$ group rat on $5 \mathrm{~d}$ before $\mathrm{ICH}$ and $1 \mathrm{~d}$ before and $2 \mathrm{~d}$ after the initiation of DOX administration from a lateral view.

$10.8 \%)$. The rats showed deficits in reaching and often failed to extend their paw to the pellet or withdraw it through a slit after grasping (Fig. 5c, Movies 1, 3). Most of the rats demonstrated a significant impairment of reaching function at $1-3 \mathrm{~d}$ after the start of DOX administration (Fig. $5 d$ ). Despite the continuous administration of DOX, the decreased success rate of ICH-FLU rats recovered gradually and the behavioral deficit disappeared at post-DOX day $7(45.1 \pm 8.3 \%$, Fig. $5 e)$. This rapid recovery would be due to compensative recruitment of other indirect descending pathways. In contrast to ICH-FLU rats, almost none of the ICH rats showed a significant decrease of reaching success rate during DOX administration ( $p>0.05$; Fig. $5 d$,e, Movies 2, 4).

In addition, the reaching movements of the rats were videotaped and analyzed with frame-by-frame observations (Fig. 5c). The reaching distance from the slit of the testing apparatus and the range of rotational movement of the wrist during reaching were calculated (Fig. $6 a-c$ ). At $2-3 \mathrm{~d}$ after DOX administration, the average reaching distance of the ICH-FLU group was significantly shortened compared with that before DOX administration (pre-DOX: $1.3 \pm 0.3 \mathrm{~mm}$; D2: $0.7 \pm 0.3 \mathrm{~mm}$; Dunnett's test: $p=0.013$; Fig. $6 d$ ). Similarly to the reaching success rate, the 
reaching distance from the slit was recovered gradually to the pre-DOX level. On the contrary, ICH rats did not show a significant alteration of reaching distance before and after DOX administration $(p>$ $0.05)$. In addition, the averaged angle of wrist pronation before grasping the pellets (Fig. 6b) was significantly decreased in the ICH-FLU group at post-DOX day 2 compared with pre-DOX (pre-DOX: $71.8 \pm$ $10.7^{\circ} ; \mathrm{D} 2: 55.5 \pm 6.3^{\circ}$; Dunnett's test: $p=$ 0.017; Fig. 6e). Similarly, the extent of supination after grasping was also decreased significantly at post-DOX day 2 compared with pre-DOX (pre-DOX: $37.3 \pm 5.0^{\circ}$; D2: $9.2 \pm 7.5^{\circ}$; Dunnett's test: $p=0.032$; Fig. $6 c, f)$. These results indicated that the range of wrist rotation during a series of reaching movement was reduced during DOX administration, but these changes were compensated for rapidly (Fig. 6e,f). The ICH control group did not show a significant change of rotational movement before and after DOX administration ( $p>0.05$; Fig. $6 e, f)$. From these data, the cortico-rubral pathway was considered to be dominantly involved in the control of proximal movements of the forelimb, such as elbow extension and wrist rotation, whereas digit movements appeared to be relatively preserved during DOX administration.

Meanwhile, the fall rate in the horizontal ladder test was not altered significantly in both groups before and during DOX administration (Dunnett's test, $p>0.05$; Fig. 6g) These results suggested that the cortico-rubral pathway was responsible for the recovery of reaching movements induced by FLU after ICH, but not for recovery of coordinated control of locomotion.

Double-infection of the cortico-rubral projection neurons was confirmed histologically by an immunohistochemical assay for EGFP expression, which was tagged to eTeNT (Fig. 5b). GFP-positive neurons were found in the motor cortex ipsilateral to the lesion (ICH: $203.1 \pm$ 71.1, ICH-FLU: $121.3 \pm 37.1$, unpaired $t$ test: $p=0.302$, Fig. $6 h$ ). The GFP-positive neurons were distributed predominantly around the RFA. The axons from these neurons could be detected in the ipsilateral red nucleus and some of them formed varicosities both in $\mathrm{RNp}$ and $\mathrm{RNm}$ (Fig. 6i). In addition, GFP-labeled fibers were located mostly in the reticular formation or partly in the cerebral peduncle rostral to the red nucleus (Fig. 6j), but there were no labeled fibers in the brainstem pyramid (Fig. 6k). These results suggested that the cortico-rubral neurons were successfully
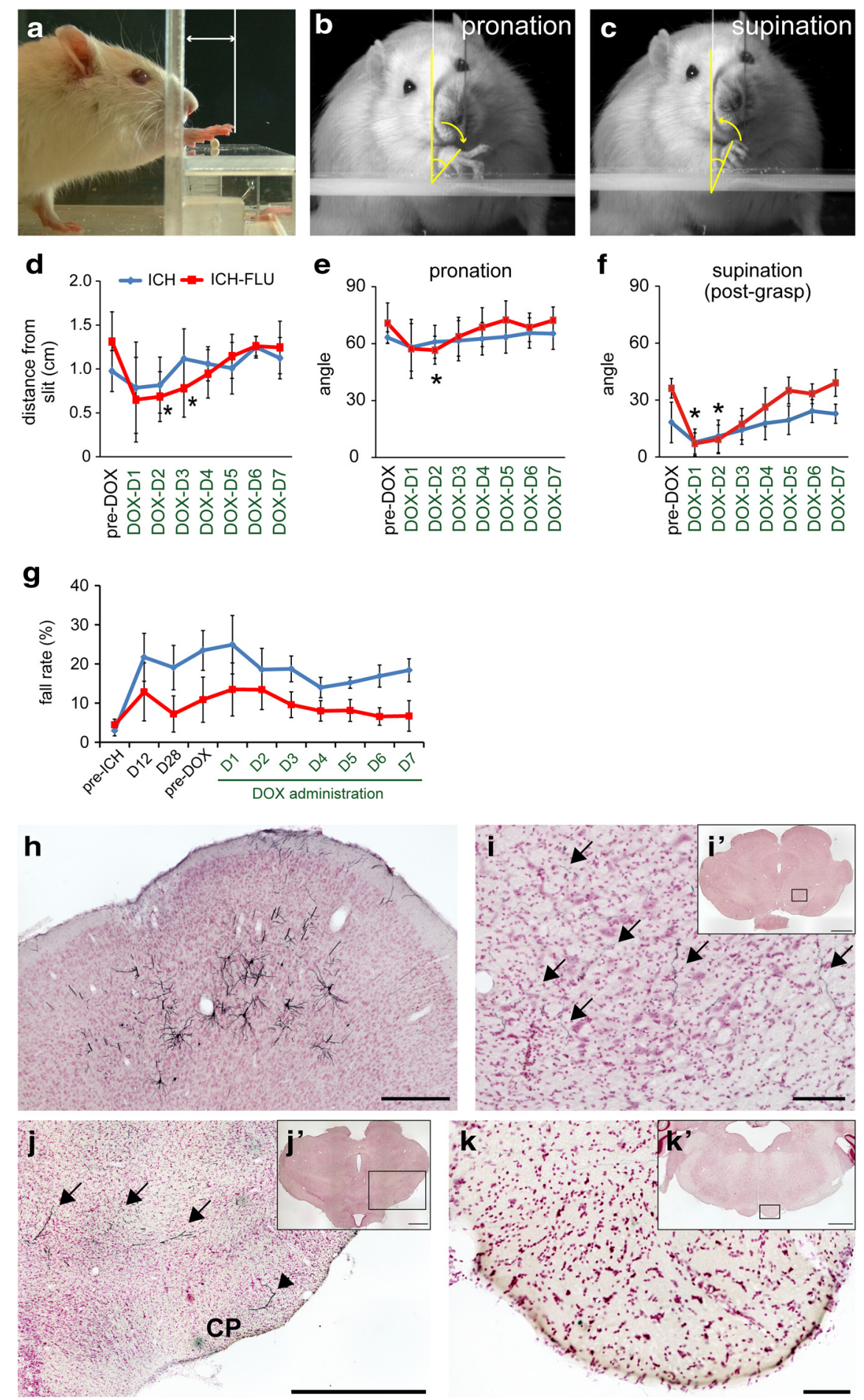

Figure 6. Detailed kinematic analyses of reaching movements and histological confirmation of the cortico-rubral neurons that were double infected by the viral vectors. $\boldsymbol{a}$-c, Frame-by-frame analysis of videotaped reaching movements was conducted for qualitative assessments. Shown are distances of reaches from the slit in the front wall of the testing apparatus $(\boldsymbol{a})$, angle of wrist pronation for grasping a pellet $(\boldsymbol{b})$, and angle of supination for withdrawal of the paw $(\boldsymbol{c}) \cdot \boldsymbol{d}$, Compared with the performance in the pre-DOX period, reaching distance from the slit was significantly shortened at $2 \mathrm{~d}$ after the initiation of D0X administration. ${ }^{*} p<$ 0.05 vs pre-DOX ICH-FLU. $\boldsymbol{e}, \boldsymbol{f}$, Range of motion of wrist pronation and supination was significantly decreased at post-D0X days 2 and $3 .{ }^{*} p<0.05$ vs pre-DOX ICH-FLU. g, Serial measurements of horizontal ladder stepping test. Neither the ICH nor the ICH-FLU group showed a significant change in fall step rate before and after DOX administration. $\boldsymbol{h}-\boldsymbol{k}$, Anti-GFP immunohistochemistry in GFP-positive pyramidal cell bodies in the ipsilesional motor cortex (h), GFP-positive axons in the ipsilesional red nucleus (i), GFP-positive axons in the reticular formation (arrows; $j$ ) and cerebral peduncle (CP: arrowhead) rostral to the red nucleus, and those in the brainstem pyramid $(\boldsymbol{k})$. $\boldsymbol{i}-\boldsymbol{k}$, Higher-magnification view of $\boldsymbol{i}^{\prime}-\boldsymbol{k}^{\prime}$. Values are shown as mean \pm SD. Scale bar, $1000 \mu \mathrm{m}$ for $\boldsymbol{j}$ and $\boldsymbol{i}^{\prime}-\boldsymbol{k}^{\prime} ; 100 \mu \mathrm{m}$ for $\boldsymbol{h}, \boldsymbol{i}$, and $\boldsymbol{k}$. 


\section{$\mathrm{ICH}$}

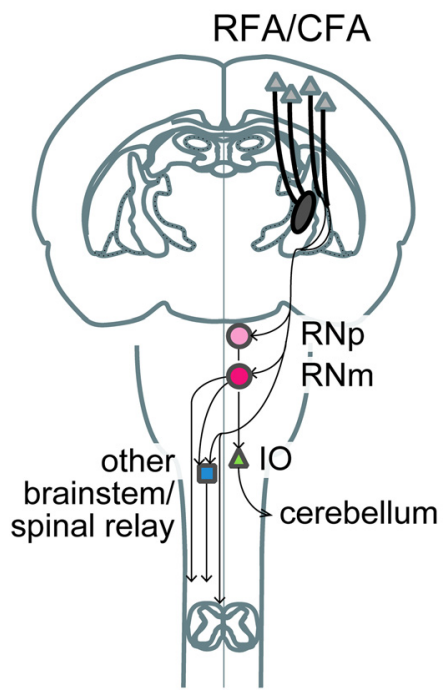

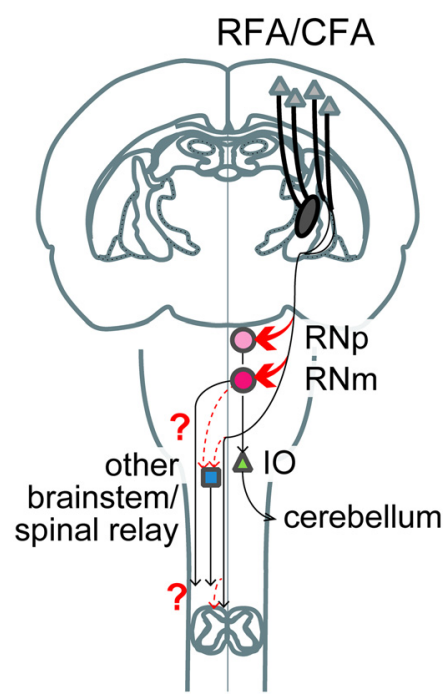

$\mathrm{ICH}-\mathrm{FLU}$

Figure 7. Schematic drawing illustrating corticofugal projections from the ipsilesional motor cortex RFA/CFAin the ICH and ICH-FLU rat. This figure does not imply that identical pyramidal neurons in the motor cortex send axons to multiple targets. After $\mathrm{ICH}$, most of the corticofugal fibers are disrupted. Intensive use of an impaired forelimb can increase cortico-rubral connections (drawn in red thick line) and might enhance transmission through the cortico-rubro-spinal pathway and the rubro-olivo-cerebellar pathway. In addition, the similar sprouting of cortco-spinal and cortico-reticular pathway (dashed red thin line; toward the other brainstem/spinal relay) might have occurred by FLU. 10, Inferior olive nucleus.

and selectively affected by combination of the two viral vectors in the present experiments, but that no or very few corticospinal axons were doubly infected. Therefore, it is likely that the impairment of the recovered forelimb movements shown in Figures 5 and 6 was caused by blockade of the cortico-rubral pathway.

\section{Discussion}

In the present study, we provided a framework to understand how the intensive use of an impaired forelimb can compensate for injured neural circuits by reorganization of the residual motor systems. Our results showed that the FLU promoted the reemergence and enlargement of the forelimb representation area in the affected motor cortex. The reemerged forelimb map of the motor cortex had substantial connections to the ipsilateral red nucleus in the ICH-FLU rats. Strikingly, the selective blockade of the connections between the motor cortex and red nucleus by using double infection of viral vectors abolished the FLU-induced recovery of forelimb function. Therefore, it was confirmed that the sprouting of cortico-rubral fibers was responsible for the functional recovery by intensive use of the impaired forelimb (Fig. 7).

\section{FLU induces cortical reorganization of the ipsilesional forelimb area of motor cortex}

Consistent with our previous reports (Ishida et al., 2011; Ishida et al., 2015), 1 week of FLU improved the recovery of forelimb function. ICH-FLU group rats were forced to rely completely on their impaired side for all daily living activities, resulting in the construction of compensatory descending motor pathways. Previously, some nonhuman primate and human studies reported that FLU and CIMT could expand the cortical motor map of the upper limb (Nudo et al., 1996; Liepert et al., 1998; Sawaki et al., 2008; Murata et al., 2015). Similarly, the present study also demonstrated expansion of the reemerged forelimb map of the affected motor cortex in ICH-FLU group. The regenerated forelimb map was positioned in the medio-caudal part of the motor cortex, which was considered to be the former vibrissa area. These results reflect the use-dependent rearrangement of the cortex after ICH. FLU might make the neurons that previously represented the vibrissa map to be involved in the control of the forelimb. Interestingly, only the FLU-treated group exhibited a forelimb map in the RFA in addition to the CFA. The forelimb area in the RFA was included in the FLU-induced reorganization and became directly responsible for the regained forelimb function because muscimol injection into the RFA clearly impaired forelimb function after recovery. In addition, GFP-positive corticorubral neurons were predominantly distributed in the RFA. Although the function of the rodent RFA remains poorly understood, it has been proposed that it may be homologous to the premotor area (PMA) or supplementary motor area (SMA) of primates (Neafsey and Sievert, 1982; Passingham et al., 1988; Rouiller et al., 1993; Eisner-Janowicz et al., 2008). The PMA and SMA are known as key regions in poststroke recovery (Liu and Rouiller, 1999; Fridman et al., 2004). Increased activity of the ipsilesional ventral PMA during the late recovery stage is related to the recovery of precision grip in monkeys after spinal cord injury (Nishimura et al., 2007) and lesion of the primary motor cortex (Murata et al., 2015). The RFA might have more plastic capacity than the CFA and may play a pivotal role in the FLU-induced reorganization of the motor system.

\section{FLU enhances plasticity in the ipsilesional motor cortex after capsular hemorrhage}

Intensive impaired limb use has been considered to cause usedependent plasticity in the CNS (Nudo et al., 1996; Murphy and Corbett, 2009). Previous reports demonstrated that early application of forced impaired forelimb use facilitated the side switching of corticospinal fibers originating from the contralesional hemisphere in the cervical cord after transection of the unilateral pyramidal tract (Maier et al., 2008). These intraspinal recrossed sprouting fibers from the intact descending tract can also be an important substrate for recovery after unilateral motor cortex infarction (Zhao et al., 2013; Wahl et al., 2014). Conversely, the present data revealed that the ipsilesional affected motor cortex and its axons descending to the red nucleus were involved in FLU-induced reorganization and recovery after ICH, which perhaps depends on the extent of damage to the corticospinal system. Contribution of the intact contralesional sensorimotor circuits would play a critical role in the recovery process after complete damage of the circuits or large stroke, whereas the residual ipsilesional circuits would be the basis of rebuilding the motor system in the case of relatively small lesion (Murphy and Corbett, 2009). Our ICH model exhibited the severe destruction of the contralateral CST, but the spared CST and most pyramidal neurons in the ipsilesional forelimb area of the motor cortex would have survived (Masuda et al., 2007; Ishida et al., 2011). Therefore, it is possible that FLU enhanced the reorganization of the ipsilesional sensorimotor cortex and corticofugal projections 
rather than that of the contralesional side in the present study. Although the detailed relationship between the effectiveness of FLU and the contribution of each side of the cortex is unknown, FLU might predominantly promote the reorganization of the ipsilesional motor cortex as a substrate for functional recovery.

\section{Plastic changes of cortico-rubro-spinal pathways is the responsible substrate for FLU-induced recovery}

The present study demonstrated that FLU caused a substantial increase of cortico-rubral connections in both RNp and RNm. In addition, GFP-positive fibers were shown in both RNp and RNm in ICH-FLU group rats. Both the cortico-RNp and cortico-RNm pathways were involved in FLU-induced plasticity. RNp projects to the inferior olive, which gives climbing fibers to the cerebellum (Allen and Tsukahara, 1974). Therefore, increased axonal sprouting in $\mathrm{RNp}$ might be related to relearning of forelimb skilled movements after ICH. Interestingly, FLU treatment also increased the sprouting in RNm. RNm is the source of the rubrospinal tract (RST), which projects to the spinal premotor network (Hongo et al., 1965, 1969) and specific populations of motoneurons involved in distal and proximal forelimb movements (Antal et al., 1992; Küchler et al., 2002). Previous studies suggested that the RST plays an alternative role to the CST in the control of proximal and distal limb movements, which enables the RST to play a compensatory role for the lesioned CST function (Martin and Ghez, 1988; Raineteau et al., 2002). Therefore, these partly newly formed cortico-rubro-spinal connections could be the principal substrate for functional recovery after stroke. The number of GFP-positive cortico-rubral neurons in the ICH-FLU rats might appear to be small (121.3 \pm 37.1$)$; however, it was found that, in cell cultures, the number of the EGFP-eTeNT fusion molecules necessary for suppressing the transmission is much smaller than that for making fluorescence visible (Ken-ichi Inoue and Masahiko Takada, personal communication). Therefore, there could be many false-negative cortical cells with synaptic transmission that was suppressed but that did not show GFP expression sufficient to be visualized. The present study demonstrated that a substantial increase of cortico-rubral connections occurred after FLU. In addition, our pathway-selective blockade experiments revealed that the cortico-rubral pathway has a causal link to the recovered reaching movements of the forelimb accompanying elbow extension and wrist rotation after ICH. Recent studies demonstrated that bilateral pyramidotomy increased, not only sprouting of the RST, but also of rubro-pontine and rubroraphe axons in mice (Ballermann and Fouad, 2006; Esposito et al., 2014; Siegel et al., 2015). The increase of cortico-rubral projections might reflect the activation of the rubrofugal descending pathway as a substitute for the injured CST. Importantly, the route involved in FLU-induced recovery might not be so simple because impaired forelimb function by selective blockade of the cortico-rubral pathway recovered gradually even during DOX administration. In addition, blockade of the cortico-rubral pathway did not affect the recovered stepping function. These data suggested the presence of other compensatory pathways. In fact, the RNm and rubrospinal tract has been considered to be degraded in humans (Nathan and Smith, 1955; Onodera and Hicks, 2010) and the present results may not be directly applied to the recovery in human patients. However, similar increase of collateral projections to other cortico-brainstem-spinal relays (see "other brainstem/spinal relays" in Fig. 7) might occur in human subjects. Intraspinal axonal sprouting from the ipsilesional and contralesional CST could also be a potent compensative route after stroke (Maier et al., 2008; Wahl et al., 2014). Due to the variability in the amount of spared CST fibers in the cervical cord in the animals used in the present study, we did not analyze intraspinal sprouting and focused instead on changes to the red nucleus. However, the present results do not exclude the possible involvement of ipsilesional and contralesional CST axons. Furthermore, in addition to the cortico-rubro-spinal pathway, previous reports have indicated that the reticulospinal tract is also involved in reorganization after a unilateral CST injury (Z'Graggen et al., 1998; Ballermann and Fouad, 2006; Zaaimi et al., 2012). The reticular formation, especially the caudal-medial part of the medullary reticular formation, is known as an essential structure for learning skilled movements (Alstermark and Isa, 2012; Esposito et al., 2014). Therefore, the possible involvement of the cortico-reticulo-spinal pathway in recovery induced by FLU should be tested in future studies.

The present study shows clearly that intensive use of an impaired forelimb induces reorganization of the injured descending route after IC hemorrhage. FLU enhances the formation of ipsilesional cortico-rubral projections, which leads to behavioral recovery. Importantly, by using the same animals throughout this study with selective loss-of-function techniques, the causal relationship between the plastic changes of the neural circuits and functional recovery was demonstrated. Clarification of the detailed mechanism of rehabilitation-induced plasticity and demonstration of its causality in recovery are essential for the establishment of better rehabilitative methods.

\section{References}

Allen GI, Tsukahara N (1974) Cerebrocerebellar communication systems. Physiol Rev 54:957-1006. Medline

Alstermark B, Isa T (2012) Circuits for skilled reaching and grasping. Annu Rev Neurosci 35:559-578. CrossRef Medline

Antal M, Sholomenko GN, Moschovakis AK, Storm-Mathisen J, Heizmann CW, Hunziker W (1992) The termination pattern and postsynaptic targets of rubrospinal fibers in the rat spinal cord: a light and electron microscopic study. J Comp Neurol 325:22-37. CrossRef Medline

Ballermann M, Fouad K (2006) Spontaneous locomotor recovery in spinal cord injured rats is accompanied by anatomical plasticity of reticulospinal fibers. Eur J Neurosci 23:1988-1996. CrossRef Medline

Biernaskie J, Corbett D (2001) Enriched rehabilitative training promotes improved forelimb motor function and enhanced dendritic growth after focal ischemic injury. J Neurosci 21:5272-5280. Medline

Dimyan MA, Cohen LG (2011) Neuroplasticity in the context of motor rehabilitation after stroke. Nat Rev Neurol 7:76-85. CrossRef Medline

Eisner-Janowicz I, Barbay S, Hoover E, Stowe AM, Frost SB, Plautz EJ, Nudo RJ (2008) Early and late changes in the distal forelimb representation of the supplementary motor area after injury to frontal motor areas in the squirrel monkey. J Neurophysiol 100:1498-1512. CrossRef Medline

Esposito MS, Capelli P, Arber S (2014) Brainstem nucleus MdV mediates skilled forelimb motor tasks. Nature 508:351-356. CrossRef Medline

Fridman EA, Hanakawa T, Chung M, Hummel F, Leiguarda RC, Cohen LG (2004) Reorganization of the human ipsilesional premotor cortex after stroke. Brain 127:747-758. CrossRef Medline

Hongo T, Jankowska E, Lundberg A (1965) Effects evoked from the rubrospinal tract in cats. Experientia 21:525-526. CrossRef Medline

Hongo T, Jankowska E, Lundberg A (1969) The rubrospinal tract. I. Effects on alpha-motoneurones innervating hindlimb muscles in cats. Exp Brain Res 7:344-364. Medline

Ishida A, Tamakoshi K, Hamakawa M, Shimada H, Nakashima H, Masuda T, Hida H, Ishida K (2011) Early onset of forced impaired forelimb use causes recovery of forelimb skilled motor function but no effect on gross sensory-motor function after capsular hemorrhage in rats. Behav Brain Res 225:126-134. CrossRef Medline

Ishida A, Misumi S, Ueda Y, Shimizu Y, Cha-Gyun J, Tamakoshi K, Ishida K, Hida H (2015) Early constraint-induced movement therapy promotes functional recovery and neuronal plasticity in a subcortical hemorrhage model rat. Behav Brain Res 284:158-166. CrossRef Medline

Jones TA, Schallert T (1994) Use-dependent growth of pyramidal neurons after neocortical damage. J Neurosci 14:2140-2152. Medline 
Kato S, Kuramochi M, Takasumi K, Kobayashi K, Inoue K, Takahara D, Hitoshi S, Ikenaka K, Shimada T, Takada M, Kobayashi K (2011) Neuron-specific gene transfer through retrograde transport of lentiviral vector pseudotyped with a novel type of fusion envelope glycoprotein. Hum Gene Ther 22:1511-1523. CrossRef Medline

Kinoshita M, Matsui R, Kato S, Hasegawa T, Kasahara H, Isa K, Watakabe A, Yamamori T, Nishimura Y, Alstermark B, Watanabe D, Kobayashi K, Isa $\mathrm{T}$ (2012) Genetic dissection of the circuit for hand dexterity in primates. Nature 487:235-238. CrossRef Medline

Küchler M, Fouad K, Weinmann O, Schwab ME, Raineteau O (2002) Red nucleus projections to distinct motor neuron pools in the rat spinal cord. J Comp Neurol 448:349-359. CrossRef Medline

Liepert J, Miltner WH, Bauder H, Sommer M, Dettmers C, Taub E, Weiller C (1998) Motor cortex plasticity during constraint-induced movement therapy in stroke patients. Neurosci Lett 250:5-8. CrossRef Medline

Liu Y, Rouiller EM (1999) Mechanisms of recovery of dexterity following unilateral lesion of the sensorimotor cortex in adult monkeys. Exp Brain Res 128:149-159. CrossRef Medline

Lois C, Hong EJ, Pease S, Brown EJ, Baltimore D (2002) Germline transmission and tissue-specific expression of transgenes delivered by lentiviral vectors. Science 295:868-872. Medline

Maier IC, Baumann K, Thallmair M, Weinmann O, Scholl J, Schwab ME (2008) Constraint-induced movement therapy in the adult rat after unilateral corticospinal tract injury. J Neurosci 28:9386-9403. CrossRef Medline

Martin JH, Ghez C (1988) Red nucleus and motor cortex: parallel motor systems for the initiation and control of skilled movement. Behav Brain Res 28:217-223. CrossRef Medline

Masuda T, Hida H, Kanda Y, Aihara N, Ohta K, Yamada K, Nishino H (2007) Oral administration of metal chelator ameliorates motor dysfunction after a small hemorrhage near the internal capsule in rat. J Neurosci Res 85:213-222. CrossRef Medline

Metz GA, Whishaw IQ (2000) Skilled reaching an action pattern: stability in rat (Rattus norvegicus) grasping movements as a function of changing food pellet size. Behav Brain Res 116:111-122. CrossRef Medline

Metz GA, Whishaw IQ (2002) Cortical and subcortical lesions impair skilled walking in the ladder rung walking test: a new task to evaluate foreand hindlimb stepping, placing, and co-ordination. J Neurosci Methods 115:169-179. CrossRef Medline

Murata Y, Higo N, Hayashi T, Nishimura Y, Sugiyama Y, Oishi T, Tsukada H, Isa $\mathrm{T}$, Onoe $\mathrm{H}$ (2015) Temporal plasticity involved in recovery from manual dexterity deficit after motor cortex lesion in macaque monkeys. J Neurosci 35:84-95. CrossRef Medline

Murphy TH, Corbett D (2009) Plasticity during stroke recovery: from synapse to behaviour. Nat Rev Neurosci 10:861-872. CrossRef Medline

Nathan PW, Smith MC (1955) Long descending tracts in man. I. Review of present knowledge. Brain 78:248-303. CrossRef Medline

Neafsey EJ, Sievert C (1982) A second forelimb motor area exists in rat frontal cortex. Brain Res 232:151-156. CrossRef Medline

Nishimura Y, Onoe H, Morichika Y, Perfiliev S, Tsukada H, Isa T (2007) Time-dependent central compensatory mechanisms of finger dexterity after spinal cord injury. Science 318:1150-1155. CrossRef Medline

Nudo RJ, Wise BM, SiFuentes F, Milliken GW (1996) Neural substrates for the effects of rehabilitative training on motor recovery after ischemic infarct. Science 272:1791-1794. CrossRef Medline

Onodera S, Hicks TP (2010) Carbocyanine dye usage in demarcating boundaries of the aged human red nucleus. PLoS One 5:e14430. CrossRef Medline
Passingham RE, Myers C, Rawlins N, Lightfoot V, Fearn S (1988) Premotor cortex in the rat. Behav Neurosci 102:101-109. CrossRef Medline

Raineteau O, Fouad K, Bareyre FM, Schwab ME (2002) Reorganization of descending motor tracts in the rat spinal cord. Eur J Neurosci 16:17611771. CrossRef Medline

Rouiller EM, Moret V, Liang F (1993) Comparison of the connectional properties of the two forelimb areas of the rat sensorimotor cortex: support for the presence of a premotor or supplementary motor cortical area. Somatosens Mot Res 10:269-289. CrossRef Medline

Sawaki L, Butler AJ, Leng X, Wassenaar PA, Mohammad YM, Blanton S, Sathian K, Nichols-Larsen DS, Wolf SL, Good DC, Wittenberg GF (2008) Constraint-induced movement therapy results in increased motor map area in subjects 3 to 9 months after stroke. Neurorehabil Neural Repair 22:505-513. CrossRef Medline

Siegel CS, Fink KL, Strittmatter SM, Cafferty WB (2015) Plasticity of intact rubral projections mediates spontaneous recovery of function after corticospinal tract injury. J Neurosci 35:1443-1457. CrossRef Medline

Sooksawate T, Isa K, Matsui R, Kato S, Kinoshita M, Kobayashi K, Watanabe D, Kobayashi K, Isa T (2013) Viral vector-mediated selective and reversible blockade of the pathway for visual orienting in mice. Front Neural Circuits 7:162. Medline

Taub E, Miller NE, Novack TA, Cook EW 3rd, Fleming WC, Nepomuceno CS, Connell JS, Crago JE (1993) Technique to improve chronic motor deficit after stroke. Arch Phys Med Rehabil 74:347-354. Medline

Ueda Y, Masuda T, Ishida A, Misumi S, Shimizu Y, Jung CG, Hida H (2014) Enhanced electrical responsiveness in the cerebral cortex with oral melatonin administration after a small hemorrhage near the internal capsule in rats. J Neurosci Res 92:1499-1508. CrossRef Medline

Wahl AS, Omlor W, Rubio JC, Chen JL, Zheng H, Schröter A, Gullo M, Weinmann O, Kobayashi K, Helmchen F, Ommer B, Schwab ME (2014) Neuronal repair: asynchronous therapy restores motor control by rewiring of the rat corticospinal tract after stroke. Science 344:1250-1255. CrossRef Medline

Wolf SL, Winstein CJ, Miller JP, Taub E, Uswatte G, Morris D, Giuliani C, Light KE, Nichols-Larsen D, Nichols-Larsen D (2006) Effect of constraint-induced movement therapy on upper extremity function 3 to 9 months after stroke: the EXCITE randomized clinical trial. JAMA 296: 2095-2104. CrossRef Medline

Xi G, Keep RF, Hoff JT (2006) Mechanisms of brain injury after intracerebral haemorrhage. Lancet Neurol 5:53-63. CrossRef Medline

Yamamoto M, Wada N, Kitabatake Y, Watanabe D, Anzai M, Yokoyama M, Teranishi Y, Nakanishi S (2003) Reversible suppression of glutamatergic neurotransmission of cerebellar granule cells in vivo by genetically manipulated expression of tetanus neurotoxin light chain. J Neurosci 23:6759-6767. Medline

Zaaimi B, Edgley SA, Soteropoulos DS, Baker SN (2012) Changes in descending motor pathway connectivity after corticospinal tract lesion in macaque monkey. Brain 135:2277-2289. CrossRef Medline

Z'Graggen WJ, Metz GA, Kartje GL, Thallmair M, Schwab ME (1998) Functional recovery and enhanced corticofugal plasticity after unilateral pyramidal tract lesion and blockade of myelin-associated neurite growth inhibitors in adult rats. J Neurosci 18:4744-4757. Medline

Zhao S, Zhao M, Xiao T, Jolkkonen J, Zhao C (2013) Constraint-induced movement therapy overcomes the intrinsic axonal growth-inhibitory signals in stroke rats. Stroke 44:1698-1705. CrossRef Medline

Zörner B, Bachmann LC, Filli L, Kapitza S, Gullo M, Bolliger M, Starkey ML, Röthlisberger M, Gonzenbach RR, Schwab ME (2014) Chasing CNS plasticity: the brainstem's contribution to locomotor recovery in rats with spinal cord injury. Brain 137:1716-1732. CrossRef Medline 